\title{
THE BLACK HOLE STABILITY PROBLEM FOR LINEAR SCALAR PERTURBATIONS
}

\author{
MIHALIS DAFERMOS AND IGOR RODNIANSKI
}

\begin{abstract}
We review our recent work on linear stability for scalar perturbations of Kerr spacetimes, that is to say, boundedness and decay properties for solutions of the scalar wave equation $\square_{g} \psi=0$ on Kerr exterior backgrounds $\left(\mathcal{M}, g_{a, M}\right)$. We begin with the very slowly rotating case $|a| \ll M$, where first boundedness and then decay has been shown in rapid developments over the last two years, following earlier progress in the Schwarzschild case $a=0$. We then turn to the general subextremal range $|a|<M$, where we give here for the first time the essential elements of a proof of definitive decay bounds for solutions $\psi$. These developments give hope that the problem of the non-linear stability of the Kerr family of black holes might soon be addressed. This paper accompanies a talk by one of the authors (I.R.) at the 12th Marcel Grossmann Meeting, Paris, June 2009.
\end{abstract}

\section{INTRODUCTION}

The stability problem for the Kerr family $\left(\mathcal{M}, g_{a, M}\right)$ of black hole solutions to the Einstein vacuum equations

$$
R_{\alpha \beta}(g)=0,
$$

the system of nonlinear hyperbolic equations governing general relativity, is one of the most important unresolved issues in the theory.

The ultimate goal would be to understand nothing less than the dynamical stability of Kerr as a family of solutions to the Cauchy problem for (1), in the spirit of the monumental monograph of Christodoulou-Klainerman [13] (see also [33]) proving the non-linear stability of Minkowski space. For the time being, however, essentially all work in the black hole case has been confined to the linearised setting. The simplest such linear problem is that of scalar perturbations, i.e. the study of solutions $\psi$ of

$$
\square_{g} \psi=0,
$$

on a fixed Kerr background $\left(\mathcal{M}, g=g_{a, M}\right)$. Equation (2) can be thought of as a poor man's substitute for the more complicated problem of gravitational perturbations, obtained by linearising (1) around the Kerr family.

The present paper will review the problem of scalar perturbations (2), in particular, the rapid recent progress concerning the Schwarzschild $a=0$ and very slowly rotating $|a| \ll M$ cases. We shall then provide, for the first time here, the essential elements of a proof of linear stability for scalar perturbations in the general subextremal case $|a|<M$, that is to say, boundedness and decay statements (see

Date: September 3, 2018. 
Theorem 1 and its corollaries) for general solutions $\psi$ of (2) with $g=g_{a, M}$ arising from arbitrary finite-energy initial data prescribed on a Cauchy surface.

In the present introductory section, we shall attempt a self-contained overview, which will be fleshed out in Sections 2-7. Sections 8-11 will then give technical details of the proof of Theorem 1 in the general $|a|<M$ case.

As there is much confusion concerning the state of affairs in this subject, we begin with a brief discussion of the classical literature.

1.1. The classical mode analysis. In the classical analysis, one does not study general solutions of (2) but rather individual modes. In the Schwarzschild case $a=0$, study of this problem was initiated in [38]. A mode is a solution of (2) of the form

$$
\psi_{m \ell}^{\omega}(r) Y_{m \ell}(\theta, \phi) e^{i \omega t}
$$

where $Y_{m \ell}$ denote the standard spherical harmonics, and $(t, r, \theta, \phi)$ denote standard Schwarzschild coordinates. In view of the stationarity and spherical symmetry of Schwarzschild, from (2) and the ansatz (3) one derives an ODE for $\psi_{m \ell}^{\omega}(r)$ in $r$, which can be rewritten in the form

$$
u^{\prime \prime}+\omega^{2} u=V u
$$

where $V$ is a nonnegative potential function, depending on $m$ and $\ell$. (Remarkably, this is also the case for gravitational perturbations [45].) It is immediate that there are no solutions of the form (3) with finite energy at $t=0$ and $\operatorname{Im}(\omega)>0$. This is the statement of 'mode stability' of Schwarzschild.

The literature on mode solutions (3) for Schwarzschild is now vast and addresses questions such as understanding transmission and reflection coefficients (relevant for black hole scattering) and locating the complex frequencies $\omega$ of so-called quasinormal modes (solutions of type (3) with $\operatorname{Im}(\omega)<0$ and finite energy on 'hyperboloidal' slices). These have been explored numerically and by methods of asymptotic analysis. See [11].

The existence of mode-type solutions for (2) on Schwarzschild-the essential starting point for this classical analysis-follows from the dimensionality of its Lie algebra of symmetries generated by the stationary Killing field and the rotations. Remarkably, as discovered by Carter, although the Kerr metric for $a \neq 0$ only has a 2-dimensional algebra of Killing fields, the wave equation (2) on Kerr still admits a non-trivial complete separation which allows one again to define mode-type solutions of the form

$$
\psi_{m \ell}^{\omega}(r) S_{m \ell}(a \omega, \phi) e^{i m \phi} e^{-i \omega t}
$$

so that $\psi_{m \ell}^{\omega}(r)$ again satisfies an ODE that can be rewritten as

$$
u^{\prime \prime}+\omega^{2} u-V(a \omega) u=0,
$$

where the potential $V$ now depends, in addition to the labels $(m, \ell)$, on the frequency $\omega$. Here, $(t, r, \theta, \phi)$ denote Boyer-Lindquist coordinates. The $S_{m \ell}$ are not explict but can be characterized as the eigenvectors of an associated operator depending on $a \omega$, which reduces to the spherical Laplacian if $a \omega=0$ (see Section 8). The above separation is related to the complete integrability of geodesic flow, discovered previously by Carter, and in fact has its origin in the existence of an additional "hidden" symmetry of the Kerr metric [44]. This separation property was extended to gravitational perturbations by Teukolsky [43]. 
For the mode analysis of scalar perturbations, the main new feature which emerges in passing from Schwarzschild to Kerr is the problem of superradiance, first discussed by Zeldovich [49]. The geometric origin of this phenomenon is simply that the 'stationary' vector field $\partial_{t}$ fails to be timelike (see Section 3.1), and thus the energy flux associated to this vector field is no longer nonnegative (see Section 7.1.1). At the level of mode analysis for $\omega \in \mathbb{R}$, this difficulty reflects itself only in the range

$$
0 \leq m \omega / a<\frac{m^{2}}{2 M\left(M+\sqrt{M^{2}-a^{2}}\right)} .
$$

It is precisely in the frequency range (5) that the sign of the energy flux through the event horizon of a solution of the form (4) is negative. The transmission and reflection coefficients of superradiant modes were probed by Starobinsky [39], who derived bounds in various asymptotic regimes. A priori, superradiance might allow for the existence of initially finite-energy solutions of type (4) with $\operatorname{Im}(\omega)>0$, and thus, growing exponentially in time. These are excluded (for both the scalar and gravitational case) in a remarkable paper of Whiting [47], who thus shows 'mode stability' of the Kerr family. Whiting's result can be viewed as the culmination of the classical mode analysis of this problem.

1.2. Linear stability? Despite the successes just outlined, the above classical mode analysis was not able to resolve what is perhaps the most basic question of linear theory, namely that of linear stability, even in the case of scalar perturbations (2). Indeed, the statement of 'mode stability' is still completely consistent with the statement that general linear perturbations $\psi$ with finite initial energy grow unboundedly in time

$$
\limsup _{t \rightarrow \infty} \psi(t, r, \theta, \phi)=\infty .
$$

The reason for the failure of mode analysis to exclude (6) can be summarised by the following:

- It is not a priori clear that solutions of (2) with finite energy can be represented as a superposition of modes with $\omega \in \mathbb{R}$.

- Even if solutions can be so represented, statements at the level of individual modes typically do not imply statements for the superposition of infinitely many modes.

Indeed, in trying to 'sum' information at the level of individual modes, one has to face certain fundamental physical obstructions which are in fact common to all wave equations in more than one spatial dimension and necessarily (see [30]) lead one to consider energy-type quantities. It is only such quantities that will identify what it is that bounds $\psi$ and excludes (6). In the black hole case, these obstructions force us to revisit to what extent we actually understand superradiance, the redshift effect and the role of solutions concentrated near trapped null geodesics, two issues which we shall discuss at length later in this paper. As we shall see, it is precisely the interaction of these difficulties which gives the present true linear stability problem its characteristic flavour.

1.3. Schwarzschild. A prototype for a true linear stability result is provided by the celebrated theorems of Wald [46] and Kay-Wald [27], which show that solutions 
$\psi$ of (2) on a Schwarzschild background remain bounded by a constant for all time, in particular, (6) does not occur:

Theorem (Kay-Wald [27]). Let $\psi$ be a solution of the wave equation on the Schwarzschild background $\left(\mathcal{M}, g=g_{M}\right)$ arising from sufficiently regular initial data on a Cauchy hyperusrface $\Sigma$. Then

$$
|\psi| \leq C \sqrt{D}
$$

in the exterior region up to and including the horizon, where $D$ is a suitable higher order energy quantity evaluated on the initial hypersurface $\Sigma .^{1}$

The proof of the above Theorem effectively initiated the study of the linear stability problem from the modern PDE point of view, according to which:

- The most fundamental boundedness statements are at the level of quantitative control of energy quantities.

- Pointwise boundedness statements follow from energy-based statements after commuting the wave equation with various vector fields, repeating the energy bounds, and applying Sobolev inequalities.

In the above, no mode-analysis need be appealed to. Such arguments are essential in proving existence theorems for non-linear wave equations, like the Einstein equations (1). From the point of view of [46, 27], linear stability away from the horizon for (2) essentially thus follows from 'standard arguments' in PDE and the fact that the stationary Killing field $\partial_{t}$ is timelike in the exterior, allowing the energy method to be applied as usual.

The more novel part of the proof of the above Theorem concerns the horizon: Since $\partial_{t}$ becomes null on the event horizon, its associated conserved energy degenerates there, and this does not allow the standard method to apply to obtain bounds on $\psi$ up to and including the horizon (see Sections 3.1 and 5.1). This difficulty was circumvented in $[46,27]$ by a number of clever, but fragile geometric arguments, exploiting in a non-trivial way the spherical symmetry but also the discrete symmetry of maximally extended Schwarzschild.

A simpler, and more robust, proof of the Kay-Wald Theorem follows by specialising [17], to be discussed below in the context of Kerr, and this new approach sheds light on what is the physical origin of the boundedness property at the horizon: it is precisely the celebrated red-shift effect, which can be quantified by exploiting in an appropriate way the generalised energy identities corresponding to a suitably-defined local-observer-based energy (see Sections 3.3, 5.3 and 6.2). This proof again does not use any decomposition of $\psi$ into either spherical harmonics or modes.

An additional linear stability statement supplementing the above Theorem would be to prove quantitative decay bounds for solutions, showing not only (7), but that $\psi$ can be bounded by a fixed decaying function whose modulus depends only on the strength of initial data. (Proving such a statement would be essential from the point of view of the question of nonlinear stability, as decay is the only known mechanism for nonlinear stability (see the proof of stability of Minkowski space $[13,33])$.) Even in the Schwarzschild case, such decay results have only become

\footnotetext{
${ }^{1}$ Otherwise, simply excluding (6) without providing such a bound would be a 'mathematical' result with no physical interpretation.
} 
available in the past few years: $[8,14,16,34,31,42]$. Proving these decay results has led in particular to better understanding of how various aspects of the underlying geometry interact in the analysis of (2), in particular, quantifying the 'obstruction' to decay caused by the presence of trapped null geodesics associated to the photon sphere (see Section 3.2). ${ }^{2}$ This latter obstruction is effectively captured by use of virial-type energy currents whose divergence is non-negative definite and degenerates precisely on such geodesics (see Section 5.2). Like the new proof [17] of boundedness, the final version of the proof of decay [16] in the Schwarzschild case takes place entirely in physical space and does not require frequency decompositions or spherical harmonics. (See Sections 5 and 6.)

1.4. The Kerr family. Up until two years ago, even the analogue of the KayWald Theorem was a completely open problem for the Kerr family, in view of superradiance and the lack of a non-negative definite conserved energy from which to start. The first result to appear concerning general solutions $\psi$ of (2), see [17], was a proof of the analogue of the Kay-Wald Theorem on a large class of underlying metrics $g$, a class which included as a special case the very slowly rotating Kerr metrics $g_{a, M}$ with $|a| \ll M$. Results giving additional decay-type statements, now specialised to the exactly Kerr case with $|a| \ll M$, rapidly followed in independent works (see Chapter 5.3 of [19] and [42]), and later also [3]. We also mention previous work concerning non-quantitative statements for azimuthal modes [23, 24]. The state of the field up to this point is described in detail in [19].

The purpose of the present paper is to review the small $|a| \ll M$ proof of [19] and complete the picture, by giving, for the first time here, the essential elements of the proof of decay results for the general subextremal case $|a|<M$. (A complete presentation, together with a proof of the refinement (13) will be forthcoming in $[21],[22]$.$) The main result is$

Theorem 1. Let $\psi$ be a solution of the wave equation $\square_{g} \psi=0$ on the Kerr background $g=g_{M, a}$ for $|a|<M$ with sufficiently regular initial data on a Cauchy hypersurface $\Sigma$, and let $\left\{\Sigma_{\tau}\right\}_{\tau \geq 0}$ be the foliation defined in Section 4.5. Then, with $|\partial \psi|^{2}$ denoting the square sum of non-degenerate derivatives as in Section 4.6, we have

- Boundedness of energy ${ }^{3}$

$$
\int_{\Sigma_{\tau}}|\partial \psi|^{2} \leq C \int_{\Sigma_{0}}|\partial \psi|^{2}
$$

- Integrated local energy decay: for arbitrary $r_{+}<R_{1}<\infty$

$$
\int_{0}^{\infty} \int_{\Sigma_{\tau} \cap\left\{r_{+} \leq r \leq R_{1}\right\}}\left(\chi|\partial \psi|^{2}+|\psi|^{2}\right) \leq C_{R_{1}} \int_{\Sigma_{0}}|\partial \psi|^{2}
$$

and

$$
\int_{\tau}^{2 \tau} \int_{\Sigma_{\tau} \cap\left\{r_{+} \leq r \leq R_{1}\right\}}|\partial \psi|^{2} \leq C_{R_{1}} D \tau^{-2} .
$$

- Polynomial-time decay of the energy-flux:

$$
\int_{\Sigma_{\tau}}|\partial \psi|^{2} \leq C D \tau^{-2}
$$

\footnotetext{
${ }^{2}$ This obstruction is not present when one restricts to finitely many spherical harmonics, as is often the case in the literature.

${ }^{3}$ Note that this energy quantity is thus non-degenerate at the horizon.
} 
Here, $\chi$ is a smooth cutoff function which vanishes in a neighborhood of the physical space projection of the trapped set, and $D$ denotes the square of an appropriate (weighted Sobolev) norm of the data, involving higher derivatives of $\psi$.

As described above, combining energy boundedness and decay with commutation arguments gives pointwise boundedness and decay statements. We give here an example of the decay statements that follow:

Corollary 1. We have the following statement of pointwise decay for $\psi$

$$
\left|r^{\frac{1}{2}} \psi\right| \leq C \sqrt{D} \tau^{-1}, \quad|r \psi| \leq C \sqrt{D} \tau^{-\frac{1}{2}} .
$$

Moreover, for any $\eta>0$

$$
|\psi| \leq C_{\eta} \sqrt{D} \tau^{-\frac{3}{2}+\eta}
$$

Before closing this introduction, we give a few comments on some of the ideas special to the Kerr case.

1.5. The interaction of the red-shift, superradiance, and trapped null orbits. We have already remarked in the Schwarzschild case the importance of understanding the red-shift effect and the role of trapped null geodesics for decay, and how these can in fact be captured by energy methods, bypassing completely mode analysis, in fact, bypassing any reference to Fourier decomposition and spherical harmonics.

Considering now the Kerr case, there are two main additional difficulties: superradiance, discussed previously, and the fact that the structure of trapped null geodesics is more complicated, at least when viewed purely in physical space. These two problems are in some sense coupled, and this coupling can be viewed as an additional, third difficulty. (See Section 7.1.) It turns out that to understand these difficulties, it is indeed useful to reconsider Carter's formal separation of $\psi$ into modes, but viewed from a slightly different perspective.

Let us note that the first issue mentioned in Section 1.2 (associated to whether general solutions $\psi$ can be written as superpositions of modes) can be sidestepped with the help of a cutoff function, by restricting consideration to a finite time slab. In such a slab, one can indeed represent a given finite-energy solution $\psi$ of (2) as a superposition of modes (4) localised at fixed frequency-triple $\omega \in \mathbb{R}, m, \ell$. If one is able to recover a suitable quantitative estimate on the solution in this timeslab, then by a bootstrap argument the estimate can easily be extended to be valid for all time.

Recall from our brief discussion of the Schwarzschild case that the key to showing quantitative bounds is constructing suitable energy currents which capture the usual energy conservation, the red-shift, and the obstruction of trapped null orbits. Key to the original boundedness result [17] on Kerr were two observations:

- In the case $|a| \ll M$, superradiant frequencies are not trapped.

- When superradiance is controlled by a small parameter, then it can be absorbed with the help of the redshift.

The two observations taken together allowed (see Section 7.2.1) an understanding of boundedness without understanding trapping. In fact, the boundedness results were obtained not just for exactly Kerr metrics but for a wide class of spacetimes whose metric is $C^{1}$-close to Schwarzschild. The decay result [19] (which is restricted to Kerr spacetimes) in the $|a| \ll M$ case replaces the first observation above with 
a complete treatment of trapping. Here the full potential of the microlocalisation provided by (4) is used: In the range of frequencies relevant for trapping, virial currents are chosen separately for each frequency-triple $(\omega, m, \ell)$, so as to degenerate at a unique value of $r$ depending on this triple. This $r$-value can be related to a null orbit with conserved quantities which are in turn associated to the frequency triple. Behind this construction is the close connection between the separation of the wave equation and the separation of the Hamilton-Jacobi equations. (See Section 7.2.2.)

The main new idea necessary for the general $|a|<M$ case is to revisit also the first observation above. In the small $|a| \ll M$ case, the fact that superradiant frequencies are not trapped follows essentially from the fact that all future-trapped null geodesics eventually leave the ergoregion. For general $|a|<M$ the latter is no longer the case. Nonetheless, remarkably,

- Superradiant frequencies are not trapped for the entire $|a|<M$ range!

In the special (marginal) case of frequency $\omega=0$, the above is related to the fact that there are no trapped null geodesics orthogonal to $\partial_{t}$. This latter fact plays a role in recent work on black hole uniqueness [1].

The above observation allows us to adapt the second observation from before to the case where superradiance is not a small parameter and still couple the conserved energy estimate, the redshift and understanding of trapping (the latter again via the microlocalisation achieved by the classical separation) so as to obtain the desired estimates (see Section 7.3).

The resolution of the linear stability problem in the $|a|<M$ case thus brings us full circle. Though based on energy methods, the proof reconnects with the classical mode analysis, now considered from a more sophisticated point of view as a tool for the 'microlocalisation' of energy currents. Moreover, the method highlights certain properties of individual modes (4), indeed of the potential functions $V(\omega)$, which do not appear to have been noticed previously, but turn out to play a fundamental part in the stability mechanism. Thus, we hope that this argument offers something new even for the reader impatient with some of the more technical difficulties of the proof.

1.6. Outlook. With Theorem 1 and its corollaries, the problem of linear stability, at least for scalar perturbations, has been essentially completely understood, leaving only the extremal $|a|=M$ case. In view of recent work of Aretakis [4] on extremal Reissner-Nordström, for extremal Kerr one in fact expects instabilities on the horizon. We should mention that electromagnetic perturbations on Schwarzschild have been treated in [5], while the problem of gravitational perturbations is the subject of recent work of Holzegel [26].

\section{The Kerr SPACE-TIME}

The detailed description of the maximally extended Kerr space-time $\left(\mathcal{M}, g_{M, a}\right)$ can be found for instance in [9],[19]. Here we simply recall the local coordinate expression of the metric in the regions which will be of interest to us. 
2.1. Boyer-Lindquist coordinates. The Kerr metric in the domain of outer communication $\mathcal{R} \subset \mathcal{M}$ (corresponding to one of the ends) is given in so-called BoyerLindquist coordinates $(t, r, \theta, \phi) \in \mathbb{R} \times\left(r_{+}, \infty\right) \times[0, \pi] \times[0,2 \pi)$ by

$$
g_{M, a}=-\frac{\Delta}{\rho^{2}}\left(d t-a \sin ^{2} \theta d \phi\right)^{2}+\frac{\rho^{2}}{\Delta} d r^{2}+\rho^{2} d \theta^{2}+\frac{\sin ^{2} \theta}{\rho^{2}}\left(a d t-\left(r^{2}+a^{2}\right) d \phi\right)^{2} .
$$

Here

$$
\Delta=r^{2}-2 M r+a^{2}, \quad \rho^{2}=r^{2}+a^{2} \cos ^{2} \theta,
$$

and $r_{+}$is the largest root of the equation $\Delta=0$,

$$
r_{ \pm}=M \pm \sqrt{M^{2}-a^{2}}
$$

The coordinate system restricted to $\theta \neq 0, \pi, \phi \neq 0$ defines an almost global chart on $\mathcal{R}$. The coordinate vectorfields $T=\partial_{t}$ and $\Phi=\partial_{\phi}$ extend to globally defined Killing fields and characterize Kerr as a stationary, axi-symmetric space-time.

Setting $a=0$, we recognize the Schwarzschild metric

$$
g_{M}=-\left(1-\frac{2 M}{r}\right) d t^{2}+\left(1-\frac{2 M}{r}\right)^{-1} d r^{2}+r^{2}\left(d \theta^{2}+\sin ^{2} \theta d \phi^{2}\right),
$$

in standard form.

It is useful to define a rescaled version of the $r$ coordinate on $r>r_{+}$by

$$
\frac{d r^{*}}{d r}=\frac{r^{2}+a^{2}}{\Delta}
$$

after suitably chosing a value for $r^{*}=0$. We will sometimes consider the $\left(t, r^{*}, \theta, \phi\right)$ coordinate system. Note that these now range in $\mathbb{R} \times \mathbb{R} \times[0, \pi] \times[0,2 \pi)$.

2.2. Kerr-star coordinates. For $|a|<M$, recall that the boundary $\partial \overline{\mathcal{R}}$ in the maximally extended Kerr spacetime $\mathcal{M}$ is a bifurcate null hypersurface $\mathcal{H}=\mathcal{H}^{+} \cup \mathcal{H}^{-}$, where $\mathcal{H}^{ \pm}$are known as the future (rsp. past) event horizons.

The geometry of $\mathcal{H}$ is not easily understood in Boyer-Lindquist coordinates, which degenerate as one approaches this hypersurface. Defining, however, so-called Kerr star coordinates $\left(r^{*}, r, \theta, \phi^{*}\right)$ on $\mathcal{R}$ by

$$
t^{*}=t+\bar{t}(r), \quad \phi^{*}=\phi+\bar{\phi}(r)
$$

where

$$
\frac{d \bar{t}}{d r}(r)=\left(r^{2}+a^{2}\right) / \Delta^{2}, \quad \frac{d \bar{\phi}}{d r}(r)=a / \Delta,
$$

then these coordinates extend regularly to be defined on a larger subset of $\mathcal{M}$, covering in particular $\mathcal{H}^{+} \backslash \mathcal{H}^{-}$, modulo the standard degeneration of the spherical coordinates on $\mathbb{S}^{2}$. In these Kerr star coordinates, the set $\mathcal{H}^{+} \backslash \mathcal{H}^{-}$now simply corresponds to the set $\left\{r=r_{+}\right\}$. We note that the Killing fields again take the form $T=\partial_{t^{*}}, \Phi=\partial_{\phi^{*}}$.

\section{Geometric phenomena of Schwarzschild and Kerr}

In this section we briefly review the geometric features of Schwarzschild and the Kerr space-time which will be particularly important for understanding the behaviour of solutions the wave equation. 
3.1. The stationary Killing field and the ergoregion. We have already remarked that for the Kerr family, the Boyer-Lindquist coordinate vector field $T=\partial_{t}$ is Killing. We consider briefly its geometry.

We begin with the Schwarzschild case. From (15), we immediately read off that $T=\partial_{t}$ is timelike everywhere in the domain of outer communications $\mathcal{R}$. On $\mathcal{H}^{+} \cup \mathcal{H}^{-}, T$ becomes null, vanishing in fact on $\mathcal{H}^{+} \cap \mathcal{H}^{-}$. (In the black hole region, $T$ becomes spacelike; this is of no concern to us here.)

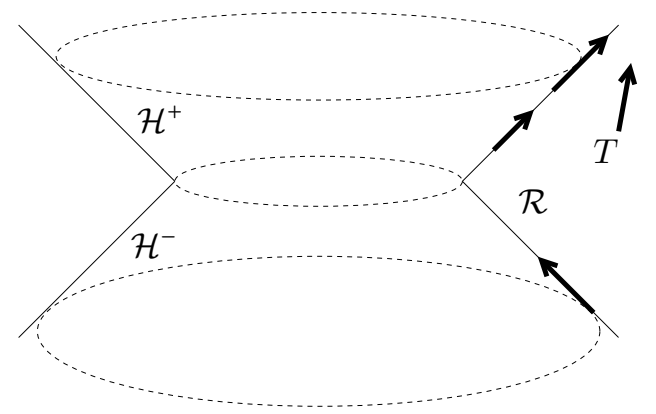

As we shall see, the fact that $T$ becomes null is already a difficulty when we discuss the notion of energy in the sections to come. In the case of the Kerr metric $g_{M, a}$ for $a \neq 0$, the situation is far worse! Now, the vectorfield $T$ fails to be everywhere timelike in the domain of outer communication $\mathcal{R}$. The subset $\mathcal{E} \subset \operatorname{int}(\mathcal{R})$ where $T$ is spacelike is known as the ergoregion. The boundary $\partial \mathcal{E}$ of $\mathcal{E}$ (in the topology of $\mathcal{R}$ ) is called the ergosphere.

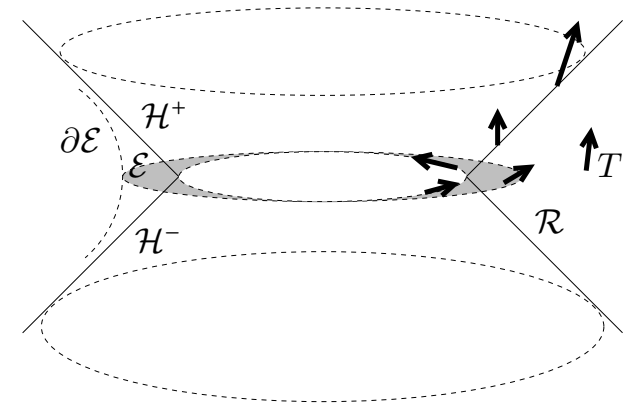

Recall also the axisymmetric Killing field $\Phi$. We note that the combination

$$
L=T+\frac{a}{r_{+}^{2}+a^{2}} \Phi
$$

is null and normal to $\mathcal{H}^{+} \backslash \mathcal{H}^{-}$. Thus $\mathcal{H}^{+} \backslash \mathcal{H}^{-}$is a Killing horizon. The Killing field $L$ is sometimes known as the Hawking vector field. We note the identity

$$
\nabla_{L} L=\kappa L
$$

where

$$
\kappa=\frac{r_{+}-r_{-}}{2\left(r_{+}^{2}+a^{2}\right)}>0 .
$$

The quantity $\kappa$ is known as the surface gravity. We note that in the extremal case $|a|=M$ (not considered here!), $\kappa$ vanishes. 
3.2. Separability of geodesic flow and trapped null geodesics. The high frequency behavior of solutions to wave equations is intimately related to the properties of null geodesic flow. We thus consider these properties in the black hole case.

Again, we begin with the Schwarzschild case $a=0$. In view of the dimensionality of the span of the Killing fields $T, \Omega_{1}, \Omega_{2}, \Omega_{3}$, it is immediate that the HamiltonJacobi equations separate, and thus, geodesic flow can be understood completely (cf. the discussion of separation of the wave equation in Section 1.1). Geodesic flow in the Schwarzschild metric is described in detail in many textbooks. One sees from the resulting equations that there are null geodesics $\gamma$ which for all affine time remain on the hypersurface $r=3 M$, the so-called photon sphere.

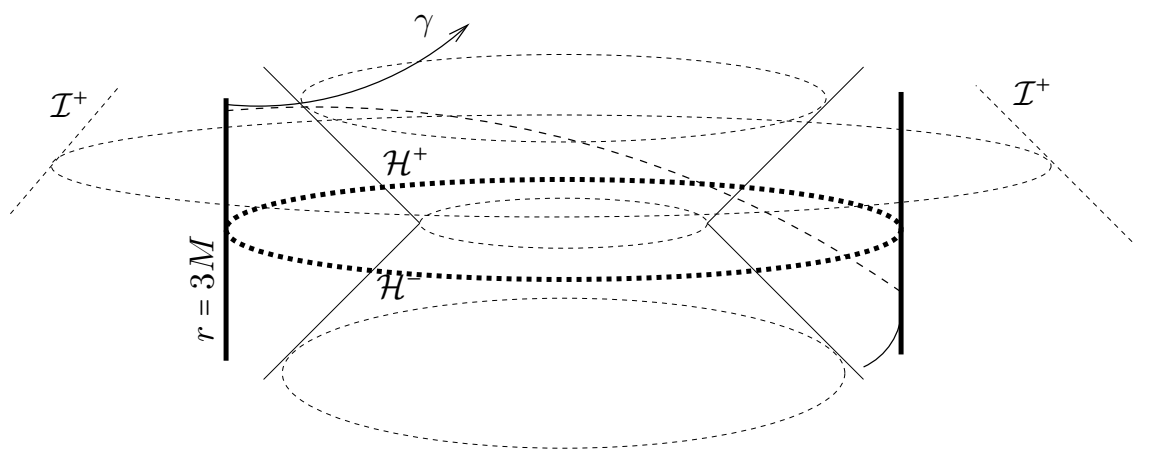

With reference to suitable asymptotic notions defining 'future null infinity' $\mathcal{I}^{+}$, we can make the following more general statement: If $\gamma(s)$ is a future-directed inextendible null geodesic in Schwarzschild $\left(\mathcal{R}, g_{M, 0}\right)$ with $\gamma(s) \in \mathcal{R} \backslash \mathcal{H}^{+}$for all $s>s_{0}$, such that moreover for all $p \in \mathcal{I}^{+}, \exists_{s}$ such that $\gamma(s) \notin J^{-}(p)$, then $\lim _{s \rightarrow \infty} r(\gamma(s))=$ $3 M$.

Turning now to the general Kerr case, remarkably, as discovered by Carter [9], geodesic flow admits, besides the conserved quantities associated to the Killing fields $T$ and $\Phi$, a third non-trivial conserved quantity (the 'Carter constant'). Thus, geodesic flow remains separable and can be completely understood. Again, this separation is related to the separation of the wave equation, discussed in Section 1.1. The dynamics is described in some detail in [11].

In contrast to the Schwarzschild case, there are now null geodesics with constant $r$ for an open range of Boyer-Lindquist- $r$ values. However, restricting to geodesics sharing a fixed triple of the nontrivial conserved quantities, there is at most one Boyer-Lindquist $r$-value, depending on the triple, to which all null geodesics neither crossing $\mathcal{H}^{+}$, nor approaching $\mathcal{I}^{+}$, must necessarily asymptote to towards the future.

3.3. Red-shift effect. An important stabilizing mechanism for the behaviour of waves near black hole event horizons is what we shall here call the "horizonlocalized" red-shift effect.

Recall that this is the red-shift relating two observers $A$ and $B$, where $B=\varphi_{\tau}(A)$ for $\tau>0$, both crossing the event horizon. In the geometric optics approximation, at horizon crossing time, the frequency of waves received by $B$ (measured with respect to proper time) from $A$ are damped (in comparison to the frequency measured by 
A) by a factor exponentially decaying in $\tau$.

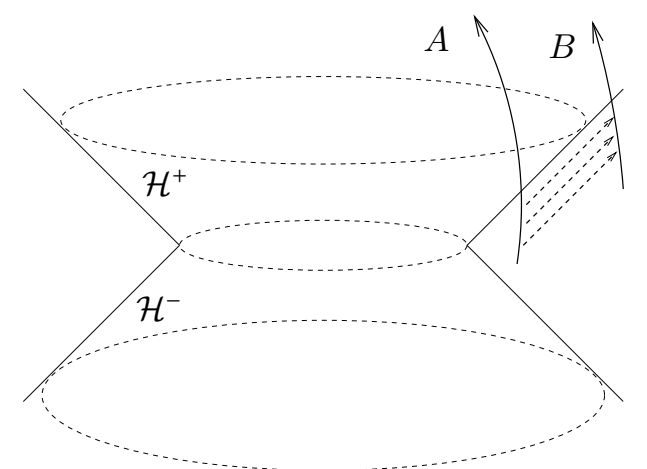

The above effect depends in fact only on the positivity of the surface gravity, as was established for non-extremal Kerr in Section 3.1. In the extremal case, we note that the above horizon-crossing time red-shift degenerates.

\section{The ENERGy Method}

In this section we introduce the machinery of compatible currents. This is a robust and invariant approach to generating $L^{2}$-based identities, which can be exploited to establish links between the geometry of space-time and the behaviour of linear (and often even nonlinear) waves.

4.1. Compatible currents in physical space. Given a Lorentzian metric $g$, let $\Psi$ be sufficiently regular and satisfy

$$
\square_{g} \Psi=F .
$$

We define

$$
\mathbf{T}_{\mu \nu}[\Psi] \doteq \partial_{\mu} \Psi \partial_{\nu} \Psi-\frac{1}{2} g_{\mu \nu} g^{\alpha \beta} \partial_{\alpha} \Psi \partial_{\beta} \Psi
$$

Given a vector field $V^{\mu}$ and a function $w$, we will define the currents

$$
\begin{gathered}
\mathbf{J}_{\mu}^{V}[\Psi]=\mathbf{T}_{\mu \nu}[\Psi] V^{\nu} \\
\mathbf{J}_{\mu}^{V, w}[\Psi]=\mathbf{J}_{\mu}^{V}[\Psi]+\frac{1}{8} w \partial_{\mu}\left(\Psi^{2}\right)-\frac{1}{8}\left(\partial_{\mu} w\right) \Psi^{2} \\
\mathbf{K}^{V}[\Psi]=\mathbf{T}_{\mu \nu}[\Psi] \nabla^{\mu} V^{\nu} \\
\mathbf{K}^{V, w}[\Psi]=\mathbf{K}^{V}[\Psi]-\frac{1}{8} \square_{g} w\left(\Psi^{2}\right)+\frac{1}{4} w \nabla^{\alpha} \Psi \nabla_{\alpha} \Psi \\
\mathcal{E}^{V}[\Psi]=-F V^{\nu} \Psi_{, v} \\
\mathcal{E}^{V, w}[\Psi]=\mathcal{E}^{V}(\Psi)-\frac{1}{4} w \Psi F
\end{gathered}
$$

Applying the divergence identity between two homologous spacelike hypersurfaces $S_{1}, S_{2}$, bounding a region $\mathcal{B}$, with $S_{2}$ in the future of $S_{1}$, we obtain

$$
\int_{S_{2}} \mathbf{J}_{\mu}^{V}[\Psi] n_{S_{2}}^{\mu}+\int_{\mathcal{B}} \mathbf{K}^{V}[\Psi]+\mathcal{E}^{V}[\Psi]=\int_{S_{1}} \mathbf{J}_{\mu}^{V}[\Psi] n_{S_{1}}^{\mu},
$$


where $n_{S_{i}}$ denotes the future directed timelike unit normal. A similar identity holds replacing $V$ by $V, w$.

4.2. Noether's theorem. The most celebrated use of (19) is in the case where $V$ is Killing, and $\Psi=\psi$ where $\psi$ satisfies $\square_{g} \psi=0$. One obtains then the conservation law

$$
\int_{S_{2}} \mathbf{J}_{\mu}^{V}[\psi] n_{S_{2}}^{\mu}=\int_{S_{1}} \mathbf{J}_{\mu}^{V}[\psi] n_{S_{1}}^{\mu} .
$$

This is a version of Noether's theorem.

4.3. The dominant energy condition. When $V$ is timelike, then one has moreover that $\mathbf{J}_{\mu}^{V}[\Psi] n_{S_{i}}^{\mu}$ is nonnegative definite, in fact

$$
\mathbf{J}_{\mu}^{V}[\Psi] n_{S}^{\mu} \geq c(S) \sum_{\alpha=0}^{3}\left|\partial_{\alpha} \Psi\right|^{2}
$$

where $\partial_{\alpha}$ denote derivatives with respect to an arbitrary system of local regular coordinates. Thus, given a timelike Killing field, (20) and (21) yield an a priori estimate for the $L^{2}$ norm on $S_{2}$ of all local derivatives of solutions $\Psi=\psi$ of the wave equation, in terms of a similar quantity evaluated on $S_{1}$.

One should not think, however, that this is the only use of identity (19). If $V$ is timelike, but not Killing, then one can estimate the bulk term

$$
\mathbf{K}^{V}[\Psi] \leq c(S) P \mathbf{J}_{\mu}^{V}[\Psi] n_{S}^{\mu}
$$

in terms of the boundary. For solutions of the wave equation, this allows one to prove that the left hand side of (19), integrated over $S_{2}(t)$ grows at most exponentially in an appropriate notion of time $t$. This is fundamental in proving local existence type results.

4.4. Virial identities. Fundamental for the considerations of the present paper will be yet another way of viewing identity (19). While in Noether's theorem and in the proof of local existence, one often considers the bulk term $\mathbf{K}^{V}[\Psi]$ as an error term, (19) is also very useful when one can show positivity properties for the bulk term $\mathbf{K}^{V}[\Psi]$. Then, provided that the boundary terms are also controlled, (19) can be used to show the boundedness of a spacetime integral quantity. This can be thought of as a weak statement of decay. This is reminiscent of the use of the virial theorem.

4.5. The hypersurfaces $\Sigma_{\tau}$. Returning now to the Kerr geometry, we shall typically apply (19) in regions bound between the event horizon, null infinity, and the leaves of a foliation associated to hypersurfaces $\Sigma_{\tau}$ to be defined below.

Let $r_{+}<r_{n}<R_{n}<\infty$. The hypersurface $\Sigma_{0}$ is constructed as follows. Define the function $f=f\left(r^{*}\right)$ according to

$$
f\left(r^{*}\right)=\int_{0}^{r^{*}} \sqrt{1-\frac{M^{2} \Delta}{\left(r^{2}+a^{2}\right)^{2}}}
$$

Then

$$
\begin{array}{lr}
\Sigma_{0}=\left\{t+f\left(r^{*}\right)=0\right\}, & \text { for } r_{+}<r<r_{n}, \\
\Sigma_{0}=\left\{t=-f\left(r_{n}^{*}\right)\right\}, & \text { for } r_{n}<r<R_{n}, \\
\Sigma_{0}=\left\{t=f\left(r^{*}\right)-\left(f\left(r_{n}^{*}\right)+f\left(R_{n}^{*}\right)\right)\right\}, & \text { for } R_{n}<r .
\end{array}
$$


Now let $\varphi_{\tau}$ denote the 1-parameter family of diffeomorphisms generated by the Killing field $T$ and define the hypersurfaces $\Sigma_{\tau}:=\varphi_{\tau}\left(\Sigma_{0}\right)$. (Equivalently, we replace everywhere $t$ by $t-\tau$ in the definition of $\Sigma_{0}$ above.)

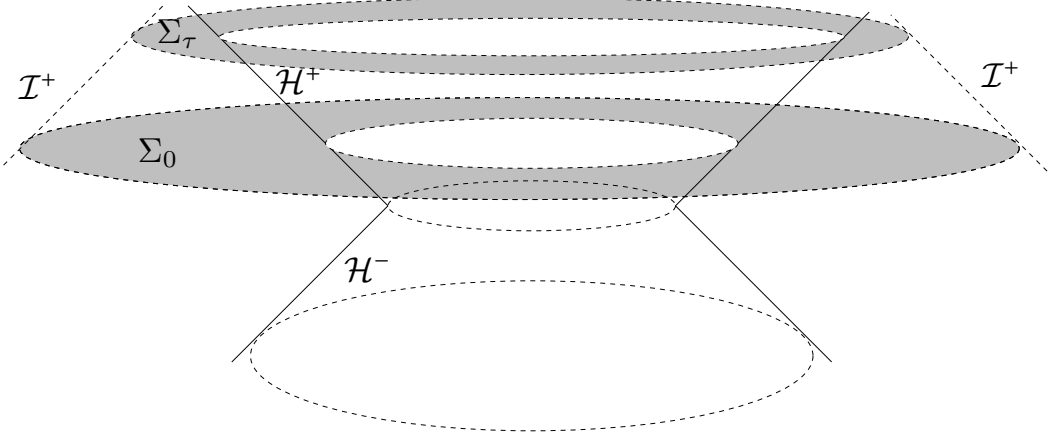

We may now denote by $\tau$ the function on $J^{+}\left(\Sigma_{0}\right)$ which is constant on the level sets $\Sigma_{\tau}$. The hypersurfaces $\Sigma_{\tau}$ are space-like:

$$
g(\nabla \tau, \nabla \tau) \leq-\frac{M^{2}-a^{2}}{2 \rho^{2}} .
$$

Near null infinity

$$
\tau=t-r^{*}+O\left(M^{2} r^{-2}\right), \quad r \rightarrow \infty
$$

while near the horizon

$$
\tau=t^{*}+O\left(M^{2}\right), \quad r \rightarrow r_{+} .
$$

By the coarea formula, we easily see that, denoting by $\mathcal{B}$ the region bounded by $\Sigma_{\tau_{1}}$ and $\Sigma_{\tau_{2}}$ and the $\mathcal{H}_{\left(\tau_{1}, \tau_{2}\right)}^{+}, \mathcal{I}_{\left(\tau_{1}, \tau_{2}\right)}^{+}$, we have that for an arbitrary function $F$,

$$
\int_{\mathcal{B}} F \sim \int_{\tau_{1}}^{\tau_{2}}\left(\int_{\Sigma_{\tau}} F\right) d \tau
$$

When the measure of integration is not denoted, the volume form is always meant. We will use this relation without comment in what follows.

4.6. Non-degenerate derivatives. Finally, since energy estimates will control various combinations of derivatives, it is useful to have suggestive notations for these.

First, we shall denote $\not$ the induced gradient on the $r=$ constant spheres.

We shall use the notation $|\partial \psi|^{2}$ to denote the type of quantity controlled by the flux of $\mathbf{J}^{N}$ through $\Sigma_{\tau}$ for $\tau \geq 0$, where $N$ is a $\varphi_{t}$-invariant vector field which is strictly timelike and $N=T$ for large $r$ (for instance, we may take the vector field of Proposition 5.3.1). Explicitly, we may define

$$
|\partial \psi|^{2}=\left(\partial_{r} \psi\right)^{2}+\left(\partial_{t^{*}} \psi\right)^{2}+|\not| \psi||^{2}
$$

for $r \leq r_{n}$, where the coordinate derivatives are interpreted in Kerr-star coordinates and

$$
|\partial \psi|^{2}=\alpha(r)\left(\partial_{t^{*}} \psi\right)^{2}+\left(\partial_{r} \psi\right)^{2}+|\not \downarrow \psi|^{2}
$$

for $r \geq r_{n}$, where $\alpha(r)$ is a positive function which vanishes as $r \rightarrow \infty$. 
Finally, let us use the notation $|\bar{\partial} \psi|^{2}$ to denote the derivatives appearing in the flux of an $N$ as above on the null hypersurfaces $\mathcal{H}^{+}$and $\mathcal{I}^{+}$, respectively. These are all tangential derivatives. We may write explicitly

$$
|\bar{\partial} \psi|^{2}=|L \psi|^{2}+|\not \psi \psi|^{2}
$$

on $\mathcal{H}^{+}$and

$$
|\bar{\partial} \psi|^{2}=|T \psi|^{2}+|\not \phi \psi|^{2}
$$

on $\mathcal{I}^{+}$

\section{SCHWARZSCHILD: THE THREE SOURCES AND COMPONENTS OF DECAY}

In this section, we describe three ingredients of the recipe for the robust derivation of the quantitative decay rates for solutions of the wave equation on Schwarzschild black hole space-times. These are directly related to the geometric phenomena studied in Section 3. We will see in the following section how these ingredients are used to show 'integrated local energy decay' and, together with an extra ingredient of a much more general type, actual decay.

The Kerr problem to be addressed later will require revisiting the strategy and, among other things, microlocalising the components of this section in a manner strongly attached to the Kerr geometry. See Section 7.

5.1. The conserved energy estimate. The Schwarzschild metric is stationary so we may apply Noether's theorem (20) for $V=T$, where $T$ is the 'stationary' Killing field. Specifically, we apply (20) to the space-time region bounded by two space-like hypersurfaces $\Sigma_{\tau_{1}}, \Sigma_{\tau_{2}}$, part of the event horizon $\mathcal{H}_{\left(\tau_{1}, \tau_{2}\right)}^{+}$and null infinity $\mathcal{I}_{\left(\tau_{1}, \tau_{2}\right)}^{+}$, i.e. we take $S_{2}=\mathcal{H}_{\left(\tau_{1}, \tau_{2}\right)}^{+} \cup \Sigma_{\tau_{2}} \cup \mathcal{I}_{\left(\tau_{1}, \tau_{2}\right)}^{+}, S_{1}=\Sigma_{\tau_{1}}$. We obtain:

$$
\int_{\Sigma_{\tau_{2}}} \mathbf{J}_{\mu}^{T}[\Psi] n_{\Sigma_{\tau}}^{\mu}+\int_{\mathcal{H}_{\left(\tau_{1}, \tau_{2}\right)}^{+}} \mathbf{J}_{\mu}^{T}[\Psi] n_{\mathcal{H}^{+}}^{\mu}+\int_{\mathcal{I}_{\left(\tau_{1}, \tau_{2}\right)}^{+}} \mathbf{J}_{\mu}^{T}[\Psi] n_{\mathcal{I}^{+}}^{\mu}=\int_{\Sigma_{\tau_{1}}} \mathbf{J}_{\mu}^{T}[\Psi] n_{\Sigma_{\tau}}^{\mu} .
$$

We shall see in Section 7.1.1 that for the general Kerr case, the above identity is a priori of limited use in view of the failure of $T$ to be timelike in the exterior. Already, however, in the case of the Schwarzschild solution with $a=0$, the vectorfield $T$, though timelike in the domain of outer communication $\mathcal{R}$, becomes null on the horizon $\mathcal{H}^{+}=\{r=2 M\}$. Thus, while all integrands in (22) are nonnegative and thus each integral taken individually is controlled by the right hand side, the first two integrands degenerate with respect to what one would have were $T$ timelike. For the first integrand, we thus only have the degenerate lower bound

$$
\mathbf{J}_{\mu}^{T}[\Psi] n_{\Sigma_{\tau}}^{\mu}(r) \geq c(r)|\partial \Psi|^{2},
$$

where $c(r)=0$ for $r=2 M$, and similarly, for the second integrand, we only have

$$
\mathbf{J}_{\mu}^{T}[\Psi] n_{\mathcal{H}^{+}}^{\mu} \geq c|T \Psi|^{2},
$$

i.e. the right hand side is missing ${ }^{4}$ the term $\left.|\not| \Psi\right|^{2}$.

From (22), we thus obtain the estimates

$$
\begin{gathered}
\int_{\Sigma_{\tau}} c(r)|\partial \psi|^{2} \leq \int_{\Sigma_{0}} \mathbf{J}_{\mu}^{T}[\Psi] n^{\mu}, \\
\int_{\mathcal{H}^{+}}|T \psi|^{2} \leq \int_{\Sigma_{0}} \mathbf{J}_{\mu}^{T}[\Psi] n^{\mu},
\end{gathered}
$$

\footnotetext{
${ }^{4}$ cf. the 'expected' $|\bar{\partial} \Psi|^{2}$
} 


$$
\int_{\mathcal{I}^{+}}|\bar{\partial} \psi|^{2} \leq \int_{\Sigma_{0}} \mathbf{J}_{\mu}^{T}[\Psi] n^{\mu}
$$

The standard approach to proving pointwise boundedness of solutions $\psi$ of (2) is by proving $L^{2}$-estimates for all derivatives of $\psi$ up to sufficiently high order. Thus, even in the Schwarzschild case, the above degeneration of (25) or (26) presents significant complications for proving stability at the horizon. See Section 6.2.

5.2. Virial estimates and trapping. For the discussion to follow here, it is useful to compare with Minkowski space. Defining a current $\mathbf{J}^{X, w}[\Psi]$ where

$$
X=f(r) \partial_{r}
$$

for specific choices of $f(r)$ and $w(r)$, one has that for suitable regions $\mathcal{B}$

$$
\int_{\mathcal{B}} \mathbf{K}^{X, w}[\Psi] \geq C_{R} \int_{\mathcal{B} \cap\{r \leq R\}}\left(|\partial \Psi|^{2}+|\Psi|^{2}\right),
$$

and thus, for solutions $\psi$ of the wave equation, identity (19) yields

$$
\int_{\mathcal{B} \cap\{r \leq R\}}\left(|\partial \psi|^{2}+|\psi|^{2}\right) \leq C\left|\int_{S_{1}} \mathbf{J}_{\mu}^{X, w}[\psi] n^{\mu}\right|+C\left|\int_{S_{2}} \mathbf{J}_{\mu}^{X, w}[\psi] n^{\mu}\right| .
$$

The above relation together with energy conservation (see Section 6.1) will already yield a weak statement of decay. This for instance already excludes stationary solutions.

It turns out that for more general metrics, trapped null geodesics are a "high frequency" obstruction for the existence of currents satisfying (29). In particular, in the Schwarzschild case, in view of the considerations of Section 3.2, one can show that there cannot exist a current $\mathbf{J}^{X, w}$ associated to a vector field $X$ of the form (28) satisfying (29) near $r=3 M$. Cf. classical results due to Ralston [37].

Remarkably, it turns out that this trapping obstruction is the only obstruction for the validity of (29) away from the horizon, and one can construct a current $\mathbf{J}^{X, w}$ where $X$ is of the form (28) satisfying

$$
\int_{\mathcal{B}} \mathbf{K}^{X, w}[\Psi] \geq C_{R_{1}, R_{2}} \int_{\mathcal{B} \cap\left\{R_{1} \leq r \leq R_{2}\right\}}\left((r-3 M)^{2}|\partial \Psi|^{2}+\left(\partial_{r} \Psi\right)^{2}+|\Psi|^{2}\right),
$$

for all $2 M<R_{1}<R_{2}<\infty$. That is to say, apart from the horizon and infinity, the control degenerates precisely on the photon sphere $r=3 \mathrm{M}$.

The vector field $X$ itself vanishes at $r=3 M$. The construction of the energy current $\mathbf{J}^{X, w}$ is quite delicate, and the original constructions in fact required spherical harmonic decompositions, a requirement that was later overcome. See $[8,14,16,7]$ and subsequent papers.

Applying now (19) in the region described in the previous section to solution $\psi$ of the wave equation (2), one obtains

$$
\begin{aligned}
& \int_{\tau_{1}}^{\tau_{2}}\left(\int_{\Sigma_{\tau} \cap\left\{R_{1} \leq r \leq R_{2}\right\}}\left((r-3 M)^{2}|\partial \psi|^{2}+\left(\partial_{r} \psi\right)^{2}+|\psi|^{2}\right)\right) d \tau \\
& \leq C_{R_{1}, R_{2}}\left(\int_{\Sigma_{\tau_{1}}}+\int_{\Sigma_{\tau_{2}}}+\int_{\mathcal{H}_{\left(\tau_{1}, \tau_{2}\right)}^{+}}+\int_{\mathcal{I}_{\left(\tau_{1}, \tau_{2}\right)}^{+}}\right) \mid \mathbf{J}_{\mu}^{X, w}[\psi] n^{\mu} .
\end{aligned}
$$


5.3. The red-shift estimate. To properly understand the behaviour of waves on the horizon, we have to take account of the red-shift effect described in Section 3.3. In the present section, we define a timelike vector field $N$ whose multiplier current $\mathbf{J}^{N}$ captures precisely this effect. This type of current was first discovered in the Schwarzschild case in [14], but it turns out, as was shown in [19], that the existence of this current is in fact a general property of stationary black hole spacetimes with Killing horizons of positive surface gravity.

In anticipation of later sections, we might as well at the onset give a statement applicable to the entire Kerr family, as the Schwarzschild case is no simpler. Theorem 7.1 of [19], specialised then to Kerr, yields

Proposition 5.3.1. Let $|a|<M, g_{a, M}$ be the Kerr metric and $\mathcal{R}$, etc., be as before. There exist positive constants $b=b(a, M)$ and $B=B(a, M)$, parameters $r_{1}(a, M)>r_{0}(a, M)>r_{+}$, and a $\varphi_{t}$-invariant timelike vector field $N=N(a, M)$ on $\mathcal{R}$ such that

(1) $\mathbf{K}^{N}[\Psi] \geq b \mathbf{J}_{\mu}^{N}[\Psi] N^{\mu}$ for $r \leq r_{0}$

(2) $-\mathbf{K}^{N}[\Psi] \leq B \mathbf{J}_{\mu}^{N}[\Psi] N^{\mu}$, for $r \geq r_{0}$

(3) $N=T$ for $r \geq r_{1}$,

where the currents are defined with respect to $g_{M, a}$.

Property 1 above can be thought to embody the exponential damping property discussed in Section 3.3.

Applying (19) with current $\mathbf{J}^{N}$ in the region of Section 5.1, we obtain using the above 'red-shift' Proposition 5.3.1 the following estimate for solutions of the homogeneous wave equation $\square_{g} \psi=0$ on Kerr:

$$
\begin{aligned}
\int_{\Sigma_{\tau_{2}}}|\partial \psi|^{2} & +\int_{\mathcal{H}_{\left(\tau_{1}, \tau_{2}\right)}^{+}}|\bar{\partial} \psi|^{2}+\int_{\mathcal{I}_{\left(\tau_{1}, \tau_{2}\right)}^{+}}|\bar{\partial} \psi|^{2} \\
& +\int_{\tau_{1}}^{\tau_{2}} \int_{\Sigma_{\tau} \cap\left\{r \leq r++s_{-}\right\}}|\partial \psi|^{2} \leq C\left(\int_{\Sigma_{\tau_{1}}}|\partial \psi|^{2}+\int_{\tau_{1}}^{\tau_{2}} \int_{\Sigma_{\tau} \cap\left\{r_{+}+s_{-} \leq r \leq r++s_{+}\right\}}|\partial \psi|^{2}\right) .
\end{aligned}
$$

Here $r_{+}+s_{-}<r_{0}$ and $r_{+}+s_{+}>r_{1}$. The red-shift effect is responsible for the horizon and the space-time term on the left hand-side of (33). It is generally effective only in a small neighborhood of the horizon. The term at null infinity appears as a consequence of $N=T$ being time-like near $\mathcal{I}^{+}$.

We note that the above estimate degenerates in the extremal case $|a|=M$, in accordance with the comments at the end of Section 3.3. This is in fact the fundamental new difficulty of the extremal problem. See [4].

The other manifestation of the red-shift effect is the following commutation statement

Proposition 5.3.2. Let $g=g_{M, a}$ and $N$ be as in Proposition 5.3.1. Then, on $\mathcal{H}^{+}$ with a distinguished generator $L$, extending $L$ to a translation invariant standard null frame $E_{1}, E_{2}, L, Y$, then for all $k \geq 0$

$$
\square_{g}\left(Y^{k} \Psi\right)=\kappa_{k} Y^{k+1} \Psi+\sum_{|\mathbf{m}| \leq k+1, m_{4} \leq k} c_{\mathbf{m}} E_{1}^{m_{1}} E_{2}^{m_{2}} L^{m_{3}} Y^{m_{4}} \Psi
$$

where $\kappa_{k}>0$. 
In the Schwarzschild case, $Y$ and $T$ together will provide around every point a timelike commutator with good properties. This is important for proving pointwise bounds. These commutations will also suffice in the $|a| \ll M$ case. In the general Kerr case, however, besides $Y$ and $T$, we shall also need to commute with the axisymmetric Killing field $\Phi$ to obtain pointwise bounds, exploiting the fact that the span of $T$ and $\Phi$ is timelike except at the horizon, where the span of $T, \Phi, Y$ again contains a timelike direction.

\section{Schwarzschild: Putting it all together}

We now proceed to show how the above components can be used to show decay type statements, beginning with integrated local energy decay.

6.1. Integrated local energy decay. The choice of the virial current $\mathbf{J}^{X, w}$ in Section 5.2 is such that the boundary terms

$$
\left(\int_{\Sigma_{\tau_{1}}}+\int_{\Sigma_{\tau_{2}}}+\int_{\mathcal{H}_{\left(\tau_{1}, \tau_{2}\right)}^{+}}+\int_{\mathcal{I}_{\left(\tau_{1}, \tau_{2}\right)}^{+}}\right)\left|\mathbf{J}_{\mu}^{X, w}[\psi] n^{\mu}\right|
$$

can be controlled (with the help also of a Hardy inequality) by

$$
\left(\int_{\Sigma_{\tau_{1}}}+\int_{\Sigma_{\tau_{2}}}+\int_{\mathcal{H}_{\left(\tau_{1}, \tau_{2}\right)}^{+}}+\int_{\mathcal{I}_{\left(\tau_{1}, \tau_{2}\right)}^{+}}\right) \mathbf{J}_{\mu}^{T}[\psi] n^{\mu}
$$

Putting together (32) and the conservation of energy expressed by (22) we thus obtain

$$
\begin{aligned}
\int_{\tau_{1}}^{\tau_{2}} & \left(\int_{\Sigma_{\tau} \cap\left\{R_{1} \leq r \leq R_{2}\right\}}\left((r-3 M)^{2}|\partial \psi|^{2}+\left(\partial_{r} \psi\right)^{2}+|\psi|^{2}\right)\right) d \tau \\
& \leq C_{R_{1}, R_{2}} \int_{\Sigma_{\tau_{1}}} \mathbf{J}_{\mu}^{T}[\psi] n^{\mu} .
\end{aligned}
$$

From this statement, one can retrieve the usual integrated local decay (away from the horizon) with a "loss of a derivative", namely:

$$
\int_{\tau_{1}}^{\tau_{2}} \int_{\Sigma_{t} \cap\left\{R_{1} \leq r \leq R_{2}\right\}}\left(|\partial \psi|^{2}+|\psi|^{2}\right) \leq C_{R_{1}, R_{2}} \int_{\Sigma_{t_{1}}}\left(\mathbf{J}_{\mu}^{T}[\psi]+\mathbf{J}_{\mu}^{T}[T \psi]\right) n_{\Sigma_{t}}^{\mu}
$$

or alternatively

$$
\int_{\tau_{1}}^{\tau_{2}} \int_{\Sigma_{t} \cap\left\{R_{1} \leq r \leq R_{2}\right\}}\left(|\partial \psi|^{2}+|\psi|^{2}\right) \leq C_{R_{1}, R_{2}} \int_{\Sigma_{t_{1}}}\left(\mathbf{J}_{\mu}^{T}[\psi]+\sum_{i=1}^{3} \mathbf{J}_{\mu}^{T}\left[\Omega_{i} \psi\right]\right) n_{\Sigma_{t}}^{\mu} .
$$

It is perhaps worth noting that if $P_{\ell} \psi$ denotes the projection of $\psi$ to the space of spherical harmonics with number $\ell$, the second term on the right hand side of (37) can be dominated by the first. Thus, we obtain precisely (35) without the degeneration at $r=3 M$. Fixed spherical harmonics thus do not see the trapping obstruction.

Finally, estimate (35) together with the red-shift estimate (33) yields the nondegenerate (cf. the degenerate (25)) energy boundedness statement

$$
\int_{\Sigma_{\tau_{2}}}|\partial \psi|^{2} \leq C \int_{\Sigma_{\tau_{1}}}|\partial \psi|^{2}
$$


and

$$
\begin{gathered}
\int_{\tau_{1}}^{\tau_{2}}\left(\int_{\Sigma_{\tau} \cap\left\{2 M \leq r \leq R_{2}\right\}}\left((r-3 M)^{2}|\partial \Psi|^{2}+\left(\partial_{r} \Psi\right)^{2}+|\Psi|^{2}\right)\right) d \tau \\
\leq C_{R_{2}} \int_{\Sigma_{\tau_{1}}}|\partial \psi|^{2}, \\
\int_{\tau_{1}}^{\tau_{2}}\left(\int_{\Sigma_{\tau} \cap\left\{2 M \leq r \leq R_{2}\right\}}\left(|\partial \psi|^{2}+\left(\partial_{r} \psi\right)^{2}+|\psi|^{2}\right)\right) d \tau \\
\quad \leq C_{R_{2}} \int_{\Sigma_{\tau_{1}}}\left(|\partial(T \psi)|^{2}+|\partial \psi|^{2}\right) .
\end{gathered}
$$

The latter statement is a true integrated local energy decay, without unnecessary degeneration at the event horizon.

6.2. Aside: boundedness without the virial identity. It is a nice exercise for the reader to see that (38) can be derived using only the red-shift estimate (33) and conservation of energy (22), i.e. without appeal to (35).

Together with the red-shift commutation Proposition 5.3.2, estimate (38) allows one to prove uniform pointwise boundedness for $\psi$ and all its derivatives to arbitrary order. See [17]. This in particular already retrieves (and strengthens) the celebrated Kay-Wald Theorem stated in the introduction.

6.3. A final ingredient: estimates at null infinity. The final ingredient for the statement of decay is a family of estimates derived in the neighborhood of future null infinity $\mathcal{I}^{+}$. These estimates have been introduced in [20] and in a way serve as a replacement of the older and less flexible approach involving the current $\mathbf{J}^{Z}$ with a vectorfield $Z$ constructed as an analog of the conformal vectorfield $Z=\left(t^{2}+r^{2}\right) \frac{\partial}{\partial t}+2 \operatorname{tr} \frac{\partial}{\partial r}$ of Minkowski space.

The family of estimates is based on the application of the current $\mathbf{J}^{V}$ with a vectorfield $V=r^{p} \frac{\partial}{\partial v}$ composed from a power of the Schwarzschild $r$-coordinate and the null coordinate vector field $\frac{\partial}{\partial v}=\frac{\partial}{\partial t}+\frac{\partial}{\partial r^{*}}$. Parameter $p$ is allowed to vary in the range $0 \leq p \leq 2$. The estimates are cleaner when $\Sigma_{\tau}$ is in fact exactly null in $r \geq R_{3}$ for some sufficiently large $R_{3}$. The resulting estimate for solutions $\psi$ of the wave equation then has the form

$$
\begin{aligned}
\int_{\Sigma_{\tau_{2}} \cap\left\{r \geq R_{3}\right\}} r^{p-2}\left|\partial_{v}(r \psi)\right|^{2}+\int_{\tau_{1}}^{\tau_{2}} \int_{\Sigma_{\tau} \cap\left\{r \geq R_{3}\right\}}\left(p r^{p-3}\left|\partial_{v}(r \psi)\right|^{2}+(2-p) r^{p-1}|\not \psi|^{2}\right) \\
\leq C\left(\int_{\Sigma_{\tau_{1}} \cap\left\{r \geq R_{3}\right\}} r^{p-2}\left|\partial_{v}(r \psi)\right|^{2}+\int_{\tau_{1}}^{\tau_{2}} \int_{\Sigma_{\tau} \cap\left\{R_{2} \leq r \leq R_{3}\right\}}|\partial \psi|^{2}+|\psi|^{2}\right),
\end{aligned}
$$

and holds with $R_{2}<R_{3}$.

6.4. Decay of the energy flux and pointwise decay. We begin with the case of Minkowski space, where already, the $p$-hierarchy of estimates (41), coupled with the statement of integrated local energy decay (30) and the energy conservation associated $\partial_{t}$, provides a new approach to the problem of robust decay.

Applying (41) first for $p=2$, and using the integrated decay estimate to control the second term on the right hand side, one may extract (by the pigeonhole principle) a suitable sequence of hypersurfaces $\Sigma_{\tau_{i}}$ for which the first term on the right hand side corresponding to the $p=1$ estimate is bounded with $\tau_{i}$ in the role 
of $\tau_{1}$. Repeating this argument and using also conservation of the usual energy, one obtains easily:

$$
\int_{\Sigma_{\tau}}|\partial \psi|^{2} \leq \frac{C}{\tau^{2}} \int_{\Sigma_{0}} r^{2}|\partial \psi|^{2}
$$

This leads, after appropriate commutation arguments, to the pointwise decay

$$
\left|r^{\frac{1}{2}} \psi\right| \leq \frac{C}{\tau}, \quad|r \psi| \leq \frac{C}{\tau^{\frac{1}{2}}}
$$

with a constant $C$ depending on the initial data. See [20].

A further refinement would also give

$$
|\psi| \leq \frac{C}{\tau^{\frac{3}{2}}} .
$$

We note that the estimate (44) matches the strongest rate of decay obtainable by the full strength of the usual vector field method. Here, however, we have avoided the use of either the conformal multiplier or commuting vector fields generating Lorentz rotations and scaling, i.e. multiplier currents or commutators with weights in $t$. This is important for the black hole case, in particular Kerr with $|a|<M .^{5}$ Moreover, in view of this additional robustness, this new method thus has an even a wider range of applications to nonlinear problems. See [48].

In the Schwarzschild case, the argument requires in addition commutation with $T$ in view of the loss of $T$ on the right hand side of (40). Other than that, it is identical to the Minkowski case! Combining (38), (40) and (41) one gets, through an analogous iteration process to the above, the decay of non-degenerate energy

$$
\int_{\Sigma_{\tau}}|\partial \psi|^{2} \leq \frac{C}{\tau^{2}} \int_{\Sigma_{0}}\left(r^{2}|\partial T T \psi|^{2}+r^{2}|\partial T \psi|^{2}+r^{2}|\partial \psi|^{2}\right) .
$$

One then obtains (43) by standard commutations. Again, one can then obtain further refinements like (44) without introducing $t$-weights.

\section{KerR: The Strategy REVISited}

In the Kerr case, we must revisit the Schwarzschild strategy in view of the new additional difficulties arising from its more complicated geometry.

\subsection{The difficulties.}

7.1.1. Superradiance. Recall the discussion of the ergoregion $\mathcal{E}$ in Section 3.1. For $p \in \mathcal{E}$, the energy density $\mathbf{J}_{\mu}^{T}[\Psi] n^{\mu}(p)$ in general fails to be nonnegative. Since if $a \neq 0, \mathcal{E} \cap \Sigma_{\tau} \neq \varnothing$ and $\mathcal{E} \cap \mathcal{H}^{+} \neq \varnothing$, it follows that the first two integrals on the left hand side of (22) no longer have positive definite integrands, and (22) no longer provides a priori control on $\Psi$.

In particular, the flux of energy to null infinity-the third term on the left hand side of (22), which is positive definite-can thus be larger than the initial energy, i.e. the term on the right hand side. This is the phenomenon of superradiance, first discussed by Zeldovich [49]. Moreover, a priori, as $\tau_{2} \rightarrow \infty$, this flux can grow without bound.

\footnotetext{
${ }^{5}$ Cf. the loss in the decay rate proportional to $a$ in the original decay result for $|a| \ll M$ in $[19]$.
} 
7.1.2. More complicated trapping. We have explained that in the presence of trapped null geodesics, considerations of geometric optics necessitate a loss of derivatives or a degeneration on the trapped set in the putative integrated local energy decay estimate. In Schwarzschild, this corresponds to the photon sphere $r=3 M$, and one could construct a current $\mathbf{J}^{X, w}$ where the vector field $X$ vanishes precisely at $r=3 M$ such that (31) holds.

As we have seen in Section 3.2 in the Kerr case for $a \neq 0$, the trapped set has a more complicated structure, which can be fully explained only in the cotangent bundle picture. Moreover, an argument in [2] shows that there does not exist a multiplier of type $\mathbf{J}^{X, w}$ satisftying an analogue of (31).

Thus, a classical vector field multiplier to capture trapping is ruled out.

7.1.3. The coupling. Recall that the estimate (40) is not used alone, but is used in conjunction with the conserved energy estimate (22). Thus, even if one can obtain a current of the form (28) satisfying a nonnegativity property generalising (31), it is not at all clear how one obtains an integrated decay estimate, in view again of superradiance, which now manifests in failure to control the boundary terms on the right hand side of (40). Thus the two difficulties are in fact coupled.

\subsection{The case $|a| \ll M$.}

7.2.1. Boundedness. The boundedness problem was resolved in [17]. At the heart of the argument were the following 2 insights regarding the difficulties of superradiance and trapping:

1. At least formally, using Carter's separation described in the Introduction, we may decompose a solution $\psi$ into its superradiant part $\psi_{b}$ and its non-superradiant part $\psi_{\sharp}$

$$
\psi=\psi_{b}+\psi_{\sharp}
$$

i.e. where $\psi_{b}$ is supported in the frequency range (5) and $\psi_{\sharp}$ in the complement of this range. ${ }^{6}$ In the case $|a| \ll M$, one easily sees that the superradiant part $\psi_{b}$ is not trapped, essentially because all future-trapped null geodesics leave the ergoregion $\mathcal{E}$. Moreover, one has a more quantitative version of this statement in that one can construct a current $\mathbf{J}^{X, w}$ satisfying an analogue of the non-degenerate (29) at least away from the horizon.

2. One can overcome the difficulty of controlling the boundary terms of a $\mathbf{J}^{X, w}$ current by the conserved energy, with the help of the red-shift. We first note that we may write

$$
\begin{aligned}
\int_{\Sigma_{\tau_{2}}}\left|\mathbf{J}_{\mu}^{T}[\psi] n_{\Sigma_{\tau}}^{\mu}\right|+\int_{\mathcal{H}_{\left(\tau_{1}, \tau_{2}\right)}^{+}}\left|\mathbf{J}_{\mu}^{T}[\psi] n_{\mathcal{H}}^{\mu}\right| & \leq \int_{\Sigma_{\tau_{2}}} \mathbf{J}_{\mu}^{T}[\psi] n_{\Sigma_{\tau}}^{\mu}+\int_{\mathcal{H}_{\left(\tau_{1}, \tau_{2}\right)}^{+}} \mathbf{J}_{\mu}^{T}[\psi] n_{\mathcal{H}}^{\mu} \\
& +\beta\left(\int_{\Sigma_{\tau_{2}}}|\partial \psi|^{2}+\int_{\mathcal{H}_{\left(\tau_{1}, \tau_{2}\right)}^{+}}|\bar{\partial} \psi|^{2}\right) \\
& \leq \int_{\Sigma_{\tau_{1}}} \mathbf{J}_{\mu}^{T}[\psi] n_{\Sigma_{\tau}}^{\mu}+\beta\left(\int_{\Sigma_{\tau_{2}}}|\partial \psi|^{2}+\int_{\mathcal{H}_{\left(\tau_{1}, \tau_{2}\right)}^{+}}|\bar{\partial} \psi|^{2}\right)
\end{aligned}
$$

\footnotetext{
${ }^{6}$ The reader may have already noticed that it is not actually the complete separation which is necessary to define this particular type of decomposition, only Fourier analysis in $t$ and $\phi$. See the comments at the end of this section. We suppress here the issue of justifying this decomposition a priori, since we discuss it in Section 9.
} 
where we have used the energy identity for $\mathbf{J}^{T}$, and the fact that $|\partial \psi|^{2},|\bar{\partial} \psi|^{2}$ contain the square sum of all derivatives appearing in the fluxes on their corresponding hypersurfaces. The last two terms in the above inequality appear precisely on the left hand side of the red-shift estimate (33). The constant $\beta$ can in fact be chosen proportional to the value of the specific angular momentum $a$ of the Kerr solution, which means that in the slowly rotating case $|a| \ll M$, given an analogue of (29) such that (34) is bounded by the right hand side of (47), we may add the analogue of (32) and (47) and a small amount of (33), and the estimates close.

The above is to be applied only to the superradiant part $\psi_{b}$, as it is only for this that we have an analogue of (29). For the non-superradiant part $\psi_{\sharp}$, we simply apply the argument of Section $6.2 .^{7}$

We obtain thus the analogue of (38) for the solution and thus we retrieve the Kay-Wald linear stability theorem, but now for Kerr with $|a| \ll M$.

Let us note finally that the above argument does not require the complete separation to define (46), but only the Fourier analysis with respect to $\partial_{t}$ and $\partial_{\phi}$. Thus, it can be adapted to the wave equation on more general axisymmetric stationary spacetimes whose metrics are $C^{1}$-close to Schwarzschild, and this is in fact the setting of [17]. ${ }^{8}$ It is interesting to note that in this very general class, one does not have uniqueness of geodesics, and thus, the behaviour of the trapped null orbits may be very different from in Schwarzschild. In particular, one does not expect to be able to prove quantitative decay results in this class.

7.2.2. Decay. For the purpose of decay, the estimate (32) is not a tool for obtaining (38) but an end in itself. We must thus obtain the analogue of this estimate for the whole solution $\psi$, not just the surperradiant projection $\psi_{b}$.

As we have seen, for this, in view of [2], one must go beyond classical multiplier currents. The property of trapped null geodesics discussed at the end of Section 3.2 strongly suggests that suitable $\mathbf{J}^{X, w}$ currents could be constructed if one were to microlocalise the construction, to distinguish between geodesics with different sets of conserved quantities, and degenerate accordingly at different values of $r$ associated to trapped null orbits, all (in the case $|a| \ll M$ !) close to $3 M$.

Again, Carter's separation ${ }^{9}$ into modes (4) suggests a particularly natural microlocalisation suitable for this problem. In fact, the existence of this separation is equivalent to the separability of geodesic flow and thus, it should naturally capture properties of geodesic flow. Using this microlocalisation we shall indeed be able to construct microlocal versions of the currents $\mathbf{J}^{X, w}$ ensuring the validity of the analogue of (31). We shall see how this is done in Sections 9-11 below.

The above microlocalisation was first carried out in Chapter 5.3 of [19]. We note that, independently, [41] showed a similar estimate in the $|a| \ll M$ case considered here, using a different microlocalisation relying on the general machinery of pseudodifferential operators. The integrability of geodesic flow is essential here too. Later, a third 'microlocalisation' was achieved in [3] by commutation with a higher order differential operator constructed from the Carter tensor. This attractive approach

\footnotetext{
${ }^{7}$ In fact, the elementary argument of Section 6.2 was first discovered in the present Kerr context [17].

${ }^{8}$ The $C^{1}$-closeness is what guarantees stability of positive-definitivity properties of nondegenerate energy currents.

${ }^{9}$ Unlike in the case of the boundedness property of Section 7.2 .1 above, here, as will be apparent we really need the complete separation.
} 
allows for a formulation of the whole argument in terms of differential (as opposed to pseudodifferential) operators, at the expense of showing results only for higher energy norms with strong weights at infinity.

Given the fact that boundedness has been proven (cf. Section 7.2.1), one need not worry about boundary terms and the difficulty of Section 7.1.3. This yields the analogue of the integrated decay statements (39), (40), where the degeneration is now on a set containing the physical space projection of the trapped set. Alternatively, one can apply directly part 2. from Section 7.2.1 to obtain the boundedness of the boundary terms, bypassing part 1 . As we shall see, however, the insight of part 1. of Section 7.2.1 will return with a vengeance in the study of the general $|a|<M$ case!

7.3. The general case $|a|<M$. The nature of geodesic flow as described in Section 3.2 suggests that 'trapping' is qualitatively similar for the whole range of $a$, and thus, microlocalised currents should again be able to capture this difficulty. This is indeed the case and is particularly easy to see with respect to the microlocalisation based on Carter's separation, as the latter is so tightly connected with the underlying geometry.

The real difficulty now, however, is trying to apply (47). For in the general case $|a|<M$, the coupling constant $\beta$ is no longer small, and thus the extra terms cannot be simply absorbed by adding a small amount of the red-shift estimate. One has to identify some non-trivial structure in the coupling of the various components.

The miracle that saves the day is the following: The insight 1. of Section 7.2.1 in fact holds for the entire $|a|<M$ range! That is to say, superradiant frequencies are not trapped, in fact, we can quantify this by saying that if the frequency is sufficiently large, one can construct a $\mathbf{J}^{X, w}$-type current with $\mathbf{K}^{X . w}$ sufficiently positive to accommodate coupling with (47) for an arbitrary $\beta$.

This miracle is somewhat unexpected because it is certainly not the case that all trapped null geodesics leave the ergoregion $\mathcal{E}$. It would be interesting to understand better the underlying reasons (if any) behind the phase-space disjointness of the trapping and superradiance phenomena. If better understood, in view of the programme of [1], this could have bearing on the uniqueness of Kerr problem.

The above remarks all concern the situation where (at least some) frequencies are large. One must also deal with the case where all frequencies are bounded without there being a large frequency parameter to exploit. In the $|a| \ll M$ case, these can be dealt with by a perturbation argument from Schwarzschild. ${ }^{10}$ Again, however, the separation gives a systematic way to deal with these difficulties which is important in the large $|a|<M$ case where perturbation arguments from Schwarzschild are not available. See Sections 10 and Section 11.7.

\section{Carter's Separation AS A GeOmetric microlocalisation}

We have given a complete overview of the proof, and now we begin with the technical details. As we have explained in the previous Section, we shall appeal

\footnotetext{
${ }^{10}$ This was the approach adopted in [19]. In our alternative approach [18], we deal with the low frequencies as in the present paper (see Section 10), precisely because the method is more systematic, can be used in the general $|a|<M$ case, and yields an independent self-contained proof of the Schwarzschild result.
} 
to Carter's separation of the wave equation, viewing it as a method to frequencylocalize energy estimates in a manner particularly suited to the local and global geometry of Kerr. In the present section, we introduce the relevant formalism.

8.1. Carter's separation. The separation of $\square_{g} \psi=0$ requires taking the Fourier transform in $t$, and then expanding into what are known as oblate spheroidal harmonics. As we shall describe in Section 9, we will apply this separation to solutions of the inhomogeneous equation

$$
\square_{g} \Psi=F,
$$

where $\Psi$ is related to $\psi$ by the application of a suitable cutoff, making $\Psi$ then $L^{2}(t)$ for each fixed $(r, \theta, \phi)$, where these denote Boyer-Lindquist coordinates on $\mathcal{R}$.

We may thus write $\Psi(t, r, \theta, \phi)$ in the form

$$
\Psi(t, r, \theta, \phi)=\frac{1}{\sqrt{2 \pi}} \int_{-\infty}^{\infty} e^{-i \omega t} \widehat{\Psi}(\omega, r, \theta, \phi) d \omega
$$

and further decompose

$$
\widehat{\Psi}(\omega, r, \theta, \phi)=\sum_{m \ell} \Psi_{m \ell}^{(a \omega)}(r) S_{m \ell}(a \omega, \cos \theta) e^{i m \phi} .
$$

The collection $\left\{S_{m \ell}(\xi, \cos \theta) e^{i m \phi}\right\}_{m \ell}$ of the eigenfunctions of the self-adjoint operator

$$
P(\xi) f=-\frac{1}{\sin \theta} \frac{\partial}{\partial \theta}\left(\sin \theta \frac{\partial}{\partial \theta} f\right)-\frac{\partial^{2} f}{\partial \phi^{2}} \frac{1}{\sin ^{2} \theta}-\xi^{2} \cos ^{2} \theta f .
$$

on $L^{2}(\sin \theta d \theta d \phi)$ forms a complete orthonormal basis on $L^{2}(\sin \theta d \theta d \phi)$. It is parametrized by a set of real discrete eigenvalues $\lambda_{m \ell}^{(\xi)}$

$$
P(\xi) S_{m \ell}(\xi, \cos \theta) e^{i m \phi}=\lambda_{m \ell}(\xi) S_{m \ell}(\xi, \cos \theta) e^{i m \phi},
$$

which have the property that

$$
\lambda_{m \ell}(\xi)+\xi^{2} \geq|m|(|m|+1) .
$$

For $\xi=0$ the oblate spheroidal harmonics $S_{m \ell}(\xi, \cos \theta) e^{i m \phi}$ reduce to the standard spherical harmonics $Y_{m \ell}$ with the corresponding eigenvalues $\lambda(0)_{m \ell}=\ell(\ell+1)$.

The usefulness of the above decomposition in the context of the wave equation on Kerr is given by the following result of Carter [10]. If $\Psi(t, r, \theta, \phi)$ satisfies

$$
\square_{g} \Psi=F,
$$

for $g=g_{a, M}$ a Kerr metric with $|a|<M$, with $\Psi(t, \cdot)$ in $L^{2}(t)$, then defining the coefficients $\Psi_{m \ell}^{(a \omega)}(r), F_{m \ell}^{(a \omega)}(r)$ as above, the following holds:

$$
\begin{aligned}
\Delta \frac{d}{d r}\left(\Delta \frac{d \Psi_{m \ell}^{(a \omega)}}{d r}\right) & +\left(a^{2} m^{2}+\left(r^{2}+a^{2}\right)^{2} \omega^{2}-\Delta\left(\lambda_{m \ell}+a^{2} \omega^{2}\right)\right) \Psi_{m \ell}^{(a \omega)} \\
& =\left(r^{2}+a^{2}\right) \Delta F_{m \ell}^{(a \omega)} .
\end{aligned}
$$

Using the definition (16) of $r^{*}$ and setting

$$
u_{m \ell}^{(a \omega)}(r)=\left(r^{2}+a^{2}\right)^{1 / 2} \Psi_{m \ell}^{(a \omega)}(r), \quad H_{m \ell}^{(a \omega)}(r)=\frac{\Delta F_{m \ell}^{(a \omega)}(r)}{\left(r^{2}+a^{2}\right)^{1 / 2}} .
$$


we obtain

$$
\frac{d^{2}}{\left(d r^{*}\right)^{2}} u_{m \ell}^{(a \omega)}+\left(\omega^{2}-V_{m \ell}^{(a \omega)}(r)\right) u=H_{m \ell}^{(a \omega)}
$$

where

$$
V_{m \ell}^{(a \omega)}(r)=\frac{4 M r a m \omega-a^{2} m^{2}+\Delta\left(\lambda_{m \ell}+\omega^{2} a^{2}\right)}{\left(r^{2}+a^{2}\right)^{2}}+\frac{\Delta\left(3 r^{2}-4 M r+a^{2}\right)}{\left(r^{2}+a^{2}\right)^{3}}-\frac{3 \Delta^{2} r^{2}}{\left(r^{2}+a^{2}\right)^{4}} .
$$

In the the Schwarzschild case:

$$
\begin{gathered}
V_{m \ell}^{(0 \omega)}(r)=(r-2 M)\left(\frac{\lambda_{m \ell}}{r^{3}}+\frac{2 M}{r^{4}}\right) \\
\left(\frac{d V}{d r^{*}}\right)_{m \ell}^{(0 \omega)}(r)=\frac{r-2 M}{r}\left(\frac{2 \lambda_{m \ell}(3 M-r)}{r^{4}}+\frac{2 M(8 M-3 r)}{r^{5}}\right) .
\end{gathered}
$$

We note that in this picture the application of $\frac{\partial}{\partial t}$ to $\Psi$ corresponds to the multiplication of $\Psi_{m \ell}^{(a \omega)}$ by $i \omega$, while $\frac{\partial}{\partial \phi}$ corresponds to the multiplication by $i m$.

8.2. Notational conventions. In what follows, let us suppress the dependence of $u, H$ and $V$ on $a \omega, m, \ell$ in our notation. ${ }^{11}$

We will also use the notation ${ }^{\prime}=\frac{d}{d r^{*}}$ and introduce a new useful non-negative parameter

$$
\Lambda=\lambda_{m \ell}(a \omega)+a^{2} \omega^{2},
$$

which according to (49) obeys

$$
\Lambda \geq|m|(|m|+1) .
$$

A word of warning: For fixed $g_{M, a}$, we shall often refer to $r^{*}$-ranges by their corresponding $r$ ranges, and functions appearing in most estimates will be written in terms of $r$. Moreover, given an $r$-parameter such as $R$, then $R^{*}$ will denote $r^{*}(R)$. It is important to remember at all times that ' always means $\frac{d}{d r^{*}}$ !

8.3. Boundary conditions. As we shall describe in Section 9, the separation process will be applied to $\Psi=\xi(\tau) \psi$ obtained by applying a cut-off $\xi$ with the property that $\xi(\tau)=1$ for $\tau \geq 1$ and $\xi(\tau)=0$ for $\tau \leq 0$ to the solution of the homogeneous wave equation $\psi$. As a consequence, the coefficients $\Psi_{m \ell}^{(a \omega)}(r)$ and hence $u_{m \ell}^{(a \omega)}(r)$ will satisfy the outgoing boundary conditions:

$$
\begin{array}{ll}
u^{\prime}+i\left(\omega-\frac{a m}{2 M r_{+}}\right) u=0, & r=r_{+}, \\
u^{\prime}-i \omega u=0, & r=\infty .
\end{array}
$$

They correspond to the standard outgoing conditions on $\Psi$

$$
\begin{array}{ll}
\left(\frac{\partial}{\partial r^{*}}-\left(\frac{\partial}{\partial t}+\frac{a}{2 M r_{+}} \frac{\partial}{\partial \phi}\right)\right) \Psi=0, & r=r_{+}, \\
\left(\frac{\partial}{\partial r^{*}}+\frac{\partial}{\partial t}\right) \Psi=0, & r=\infty .
\end{array}
$$

\footnotetext{
${ }^{11}$ Before suppressing the dependence, it might be useful to remark that in fact, the functions $u, H, V$, etc., depend on both $a \omega$ and $a$, whereas the parameters $\lambda_{m \ell}$ depend only on $a \omega$. For the former 3 , will view reference to the $a$-dependence as implicit in the reference to $a$ in $a \omega$. Hence, our writing $0 \omega$ instead of 0 in (52).
} 
We note that the vectorfield $L=\frac{\partial}{\partial t}+\frac{a}{2 M r_{+}} \frac{\partial}{\partial \phi}$ is the distinguished null generator of the future horizon $\mathcal{H}^{+}$mentioned, for instance, in the earlier discussion of the red-shift effect and surface gravity.

8.4. The separated current templates. To describe the analogue of energy currents localized to frequency triplet $(\omega, m, \ell)$, it will be convenient to define the following current templates.

8.4.1. The microlocal $\mathbf{J}^{T}$. We first define the microlocal analogue of the conserved energy current, i.e. the $\mathbf{J}^{T}$ current:

$$
Q_{T}[u]=\omega \operatorname{Im}\left(u^{\prime} \bar{u}\right)
$$

We compute

$$
Q_{T}^{\prime}=\omega \operatorname{Im}(H \bar{u})
$$

We observe that the boundary conditions (54), (55) imply that

$$
Q_{T}\left(r_{+}\right)=-\omega\left(\omega-\frac{a m}{2 M r_{+}}\right)|u|^{2}, \quad Q_{T}(\infty)=\omega^{2}|u|^{2}
$$

and that $Q_{T}(\infty)$ and $-Q_{T}\left(r_{+}\right)$are both positive in the non super-radiant regime

$$
\frac{m \omega}{a} \notin\left[0, \frac{m^{2}}{2 M r_{+}}\right] \text {. }
$$

8.4.2. The microlocal virial currents $\mathbf{J}^{X, w}$. Next, we define the microlocal analogue of the virial currents $\mathbf{J}^{X, w}$ where $X$ is in the direction of $\partial_{r^{*}}$, and $w$ is a suitable function.

It is most natural to parametrize such choices of currents as follows. For arbitrary functions $f\left(r^{*}\right), h\left(r^{*}\right), y\left(r^{*}\right)$, we define the currents

$$
\begin{aligned}
& Q_{0}^{f}=f\left[\left|u^{\prime}\right|^{2}+\left(\omega^{2}-V\right)|u|^{2}\right]+f^{\prime} \operatorname{Re}\left(u^{\prime} \bar{u}\right)-\frac{1}{2} f^{\prime \prime}|u|^{2}, \\
& Q_{1}^{h}=h \operatorname{Re}\left(u^{\prime} \bar{u}\right)-\frac{1}{2} h^{\prime}|u|^{2} \\
& Q_{2}^{y}=y\left[\left|u^{\prime}\right|^{2}+\left(\omega^{2}-V\right)|u|^{2}\right] .
\end{aligned}
$$

We note the simple relation: $Q_{0}^{f}=Q_{1}^{f^{\prime}}+Q_{2}^{f}$. We compute

$$
\begin{aligned}
& \left(Q_{0}^{f}\right)^{\prime}=2 f^{\prime}\left|u^{\prime}\right|^{2}-f V^{\prime}|u|^{2}-\frac{1}{2} f^{\prime \prime \prime}|u|^{2}+2 f \operatorname{Re}\left(u^{\prime} \bar{H}\right)+f^{\prime} \operatorname{Re}(u \bar{H}), \\
& \left(Q_{1}^{h}\right)^{\prime}=h\left[\left|u^{\prime}\right|^{2}+\left(V-\omega^{2}\right)|u|^{2}\right]-\frac{1}{2} h^{\prime \prime}|u|^{2}+h \operatorname{Re}(u \bar{H}) \\
& \left(Q_{2}^{y}\right)^{\prime}=y^{\prime}\left[\left|u^{\prime}\right|^{2}+\left(\omega^{2}-V\right)|u|^{2}\right]-y V^{\prime}|u|^{2}+y \operatorname{Re}\left(u^{\prime} \bar{H}\right) .
\end{aligned}
$$

8.4.3. The microlocal red-shift current $\mathbf{J}^{N}$. Finally, we define the microlocal analogue of the red-shift current $\mathbf{J}^{N}$. This takes the form

$$
Q_{\text {red }}^{z}=z\left[\left|u^{\prime}+i\left(\omega-\frac{a m}{2 M r_{+}}\right) u\right|^{2}+\left(\omega^{2}-V-\left|\omega-\frac{a m}{2 M r_{+}}\right|^{2}\right)|u|^{2}\right],
$$

where $z$ will be chosen to be a suitable function blowing up as $r \rightarrow r_{+}$.

For convenience we denote

$$
\omega_{+}:=\frac{a m}{2 M r_{+}}
$$

a constant which can be also characterized by the relation

$$
\omega^{2}-V\left(r_{+}\right)=\left|\omega-\omega_{+}\right|^{2} .
$$


Let

$$
\tilde{V}:=V+\left|\omega-\omega_{+}\right|^{2}-\omega^{2}
$$

so that $\tilde{V}\left(r_{+}\right)=0$ and $\tilde{V}^{\prime}(r)=V^{\prime}(r)$. We then compute

$$
\left(Q_{\mathrm{red}}^{z}\right)^{\prime}=z^{\prime}\left|u^{\prime}+i\left(\omega-\omega_{+}\right) u\right|^{2}-(z \tilde{V})^{\prime}|u|^{2}+2 z \operatorname{Re}\left(u^{\prime} \bar{H}\right) .
$$

\section{Proof of Theorem 1}

In the rest of this paper, we give detailed account of the proof of Theorem 1.

9.1. The continuity argument in $a$. The proof of Theorem 1 proceeds via a continuity argument in the Kerr parameter $a$. This is central to the logic of the proof because we are relying (recall Section 8.1) on taking the Fourier transform in time, and this requires some a priori information.

We must show thus that the set of $a$ for which the statement of Theorem 1 holds (with a constant $C=C(a)$ whose form is given by the proof) is nonempty, and both open and closed in the subset topology of $(-M, M)$. We note that in principle, the function $C(a)$ will diverge as $|a| \rightarrow M$.

The value $a=0$ corresponds to Schwarzschild. The validity of the result in the Schwarzschild case thus satisfies the requirement on non-emptiness. (In fact, the considerations of Section 10 below can be used to provide an independent, selfcontained proof for the Schwarzschild case. See [18].)

9.2. Openness. The template for the openness argument is actually the small $|a| \ll M$ case, treated in detail in [18], which can be thought of as implementing the openness argument in the vicinity of $a=0$.

The actual openness argument is an amalgation of techniques from the closedness argument and the small $a$ case. For convenience, here we shall treat the openness case as if it were exactly the small $a$ case. This will allow us also to review in more detail this special case.

9.2.1. Cutoffs. For openness, we do not know that $\psi$ decays a priori in time, and thus, our cutoff $\xi$ must be supported only in $0 \leq \tau \leq \tau_{f}$ for some large $\tau_{f}$, where $\xi=1$ in the range $1 \leq \tau \leq \tau_{f}-1$ say.

By a bootstrap argument, $\tau_{f}$ will finally be taken to infinity. The proximity to something known (inherent in proving openness) will be essential here. See Section 9.2.3.

9.2.2. A microlocalised virial current. For convenience, let us actually restrict to the $|a| \ll M$ case in our discussion of this construction, because it is a nice illustration of how proximity to a solution where estimates have already been established can be used. (The general openness will follow from an amalgamation of the ideas here and in the closedness section.)

We have discussed in Section 7.2.2 that we need a microlocal version of the current $\mathbf{J}^{X, w}$ and of the positivity statement which schematically we write

$$
\int \mathbf{K}^{X, w}[\Psi] \geq b \int_{R_{0}}^{R_{1}}\left|\partial_{r^{*}} \Psi\right|^{2}+\chi|\partial \Psi|^{2}+|\Psi|^{2}-\int \mathcal{E}^{X, w}[\Psi] .
$$

where $r_{+}<R_{0}<3 M<R_{1}<\infty$. The $\mathcal{E}^{X, w}[\Psi]$ occurs (recall (19)) from the fact that $\Psi=\xi \psi$ and thus satisfies an inhomogeneous wave equation. 
In the formalism of our separation, this means that for various frequency ranges $(\omega, m, \ell)$, by employing various compositions of the virial microlocalised currents $Q_{0}^{f}, Q_{1}^{h}, Q_{2}^{y}$, we seek a current $Q$ such that

$$
\int_{-\infty}^{\infty} Q^{\prime}[u] \geq \int_{R_{0}^{*}}^{R_{1}^{*}}\left|u^{\prime}\right|^{2}+\chi\left(\lambda+\omega^{2}\right)\left|u^{2}\right|+|u|^{2}-\int_{-\infty}^{\infty} H \cdot(f, h, y) \cdot\left(u, u^{\prime}\right),
$$

where the last term is schematic for various combinations (such as $f \operatorname{Re}\left(u^{\prime} \bar{H}\right)$ ) which occur. The terms $Q, u, \chi, \lambda, H, f, h, y$ should all be understood with the full set of indices, i.e. $u_{m \ell}^{(a \omega)}$, etc., here omitted for brevity. For frequencies for which trapping is relevant, $\chi$ will vanish at a unique value of $r$ associated with the frequency triple. One restricts the domain of integration on the first term on the right hand side because one expects this virial current not to control things at the horizon and infinity.

We may rewrite (57) above as an estimate

$$
b \int_{R_{0}^{*}}^{R_{1}^{*}}\left|u^{\prime}\right|^{2}+\chi\left(\lambda+\omega^{2}\right)\left|u^{2}\right|+|u|^{2} \leq \int_{-\infty}^{\infty} Q^{\prime}[u]+\left|\int_{-\infty}^{\infty} H \cdot(f, h, y) \cdot\left(u, u^{\prime}\right)\right|,
$$

and thus

$$
b \int_{R_{0}^{*}}^{R_{1}^{*}}\left|u^{\prime}\right|^{2}+\chi\left(\lambda+\omega^{2}\right)\left|u^{2}\right|+|u|^{2} \leq Q[\infty]-Q[-\infty]+\left|\int_{-\infty}^{\infty} H \cdot(f, h, y) \cdot\left(u, u^{\prime}\right)\right|
$$

9.2.3. Summing. Restoring now the indices, summing (58) over $m$ and $\ell$ and integrating over $\omega$, we obtain by Plancherel for $r_{c} \leq R_{0}<R_{1} \leq R_{c}$

$$
\begin{aligned}
b \int_{0}^{\tau_{f}} d \tau \int_{\Sigma_{\tau} \cap\left\{r \in\left[R_{0}, R_{1}\right]\right\}}\left(\chi|\partial \psi|^{2}+\left|\partial_{r^{*}} \psi\right|^{2}+|\psi|^{2}\right) & \leq \int_{\mathcal{H}^{+}}\left|\mathbf{J}_{\mu}^{T} n_{\mathcal{H}}^{\mu}\right|+\int_{\mathcal{I}^{+}} \mathbf{J}_{\mu}^{T} n_{\mathcal{I}}^{\mu} \\
& +\int_{\Sigma_{0}}|\partial \psi|^{2}+\int_{\Sigma_{\tau_{f}-1}}|\partial \psi|^{2} .
\end{aligned}
$$

Here, $\chi$ now denotes a smooth cut-off function which vanishes on a small ( $a$-size) neighborhood of $r=3 M$.

Below we explain the main features of how we obtained (59):

1. The range of $\tau$-integration on the left hand side of $(72)$ is restricted to $\left[0, \tau_{f}\right]$. This is due to the fact that our microlocal analysis was applied to the function $\Psi=\xi(\tau) \psi$ with a cut-off function $\xi$ vanishing for $\tau \leq 0, \tau \geq \tau_{f}$. The former property of $\xi$ led to the microlocal outgoing boundary conditions (54), (55).

2. The boundary terms on $\mathcal{H}^{+}$and $\mathcal{I}^{+}$arise from the corresponding boundary terms $Q( \pm \infty)$ in the microlocal virial inequalities. The fact that these terms are controlled by the $\mathbf{J}^{T}$ fluxes is a consequence of the boundary conditions (54), (55). For instance,

$$
Q_{0}^{f}(+\infty)=f\left(\left|u^{\prime}\right|^{2}+\left(\omega^{2}-V\right)|u|^{2}\right)(+\infty)=2 f \omega^{2}|u|^{2}(+\infty)=i f \omega \bar{u}\left(-i \omega u-u^{\prime}\right)(+\infty),
$$

which by Plancherel can easily be seen to be bounded by the $\mathbf{J}^{T}$-flux upon summation.

3. The contribution of the inhomogeneous term $H$ in (58) gives rise to the terms integrated over $\Sigma_{0}$ and $\Sigma_{\tau_{f}-1}$ on the right hand side of (59). Let us see briefly how this occurs.

Recall that $H$ arises from the term $F=\square_{g} \xi \psi+2 \nabla^{\mu} \xi \nabla_{\mu} \psi$, introduced by the cut-off $\xi$ mutliplied to $\psi$, and that $F$ is thus supported in $\tau \in[0,1] \cup\left[\tau_{f}-1, \tau_{f}\right]$. 
For concreteness, consider a term

$$
\int f_{m \ell}^{(a \omega)}(r) \operatorname{Re}\left(\frac{d u_{m \ell}^{(a \omega)}}{d r^{*}} \bar{H}_{m \ell}^{(a \omega)}\right)
$$

Were this integral in fact restricted to $\left[R_{0}, R_{1}\right]$, it is easy to see that it can be controlled by a little bit of the left hand side of (59) and the last two terms on the right hand side. For we apply Cauchy-Schwarz to bound this term by

$$
\int\left|f_{m \ell}^{(a \omega)}(r)\right|\left(\left|\frac{d u_{m \ell}^{(a \omega)}}{d r^{*}}\right|^{2}+\epsilon^{-1}\left|H_{m \ell}^{(a \omega)}\right|^{2}\right),
$$

and applying Plancherel, one can then absorb the first term into the left hand side, while the right hand side, together with a finite time energy estimate and the above comment on the support of $F$, can easily be seen to be bounded by the claimed boundary terms.

On the complement of $\left[R_{0}, R_{1}\right]$, one needs a slightly different strategy, which takes into account that the term $H_{m \ell}^{(a \omega)}(r)$ arises from the compactly supported expression $F=\square_{g} \xi(\tau) \Psi+2 \nabla^{\mu} \xi(\tau) \nabla_{\mu} \Psi$ with a smooth cut-off function $\xi(\tau)=1$ for $\tau \geq 1$ and $\xi(\tau)=0$ for $\tau \leq 0$. In [18], we have arranged our mutipliers, i.e., function $f_{m \ell}^{(a \omega)}(r)$ in this example to be independent of the parameters $\omega, m, \ell$ outside of $\left(R_{0}, R_{1}\right)$ so that after summation in $m, \ell$ and integration in $\omega$ we obtain the term

$$
\int_{0}^{\infty} d \tau \int_{\Sigma_{\tau} \cap\left\{r \notin\left(R_{0}, R_{1}\right)\right\}} \frac{d \Psi}{d r^{*}} F .
$$

Since $F$ is supported in the interval $\tau \in(0,1)$ we can easily relate the above expression to the initial data. More generally, the basic idea for treating compactly supported in $\tau$ inhomogeneous terms in the equation $\square_{g} \psi=F$ is to couple them with the $\mathbf{J}^{T}$ based energy estimate for $\psi$. (The precise analog of this for the wave equation in Minkowski space is that the integrated local energy decay estimate can have two incarnations (with arbitrary $\alpha>0$ )

$$
\begin{aligned}
& b\left(\left\|\frac{\partial \psi}{\langle r\rangle^{\frac{1}{2}+\alpha}}\right\|_{L_{t}^{2} L_{x}^{2}}+\left\|\frac{\psi}{\langle r\rangle^{\frac{3}{2}+\alpha}}\right\|_{L_{t}^{2} L_{x}^{2}}\right) \leq\left\|\partial \psi_{0}\right\|_{L_{x}^{2}}+\left\|\langle r\rangle^{\frac{1}{2}+\alpha} F\right\|_{L_{t}^{2} L_{x}^{2}}, \\
& b\left(\left\|\frac{\partial \psi}{\langle r\rangle^{\frac{1}{2}+\alpha}}\right\|_{L_{t}^{2} L_{x}^{2}}+\left\|\frac{\psi}{\langle r\rangle^{\frac{3}{2}+\alpha}}\right\|_{L_{t}^{2} L_{x}^{2}}\right) \leq\left\|\partial \psi_{0}\right\|_{L_{x}^{2}}+\|F\|_{L_{t}^{1} L_{x}^{2}}
\end{aligned}
$$

and the second estimate is more advantageous if $F$ has compact support in $t$.)

These technical issues are carefully treated in [18] and we proceed without making any further comments.

9.2.4. Completing the argument. We may now couple (59) to (47), and, applying the red-shift estimate, as described in Section 7.2.1, we may obtain

$$
\int_{0}^{\tau_{f}} d \tau \int_{\Sigma_{\tau} \cap\left\{r \leq R_{1}\right\}}\left(\chi|\partial \psi|^{2}+|\psi|^{2}\right) \leq C \int_{\Sigma_{0}}|\partial \psi|^{2}
$$

where we are using that we may treat $\beta$ as a small parameter to absorb terms. The cutoff time was arbitrary and does not appear in the constant, and thus, we may finally replace $\tau_{f}$ with $\infty$.

We have thus reduced openness to showing (58) for all frequency ranges. This will be done explicitly in Section 10 for $|a| \ll M$, giving us the opportunity to review this simpler case-treated in full detail in [18]-first. As said earlier, the actual 
openness argument for the general case can be implemented by an amalgamation of these multipliers and the types of multipliers we shall use in the closedness case. Thus, the real new difficulties of the general $|a|<M$ case concern closedness.

9.3. Closedness. For closedness, it suffices to improve an estimate for a solution already known to decay, i.e. it suffices to consider a sequence of $a_{i} \rightarrow a$ for which the statement of Theorem 1 holds, and to show that the constant $C\left(a_{i}\right)$ of the theorem can be uniformly bounded as $a_{i} \rightarrow a$.

9.3.1. Cutoffs. In view of the fact that we already know that the solution is integrable towards the future, we need only cutoff near the initial data surface $\Sigma_{0}$. That is to say, we apply a cutoff $\xi$ to the solution $\psi$ of (2) such that $\xi=1$ for $\tau \geq 1$ and $\xi=0$ for $\tau \leq 0$ and we then apply the separation of Section 8.1 to $\Psi=\xi \psi$. In contrast to the openness case, in view of the new cutoff, the inhomogeneous term $H_{m \ell}^{(a \omega)}(r)$ can be thought to arise solely from initial data for $\psi$ on $\Sigma_{0}$.

9.3.2. A microlocalised combined current. Whereas in the small $|a| \ll M$ case, we needed merely to microlocalise the construction of $\mathbf{J}^{X, w}$, and the boundary terms could be handled using (47) and the smallness of the parameter $\beta$, here, the coupling between the virial current, the problem of superradiance, and the red-shift is nontrivial. To exploit the structure described in Section 7.3, all currents must be microlocalised. Essentially, we would like a microlocal version of the inequalities

$$
\begin{gathered}
\int \mathbf{K}^{X, w}[\Psi]+\mathbf{K}^{\alpha N}[\Psi]+\mathbf{K}^{\beta T}[\Psi] \geq \int\left|\partial_{r^{*}} \Psi\right|^{2}+\chi|\partial \Psi|^{2}+\Psi^{2}-\int \mathcal{E}^{X, w}[\Psi], \\
\int_{\mathcal{H}^{+} \cup \mathcal{I}^{-}}\left(\mathbf{J}_{\mu}^{X, w}+\beta \mathbf{J}_{\mu}^{N}+\alpha \mathbf{J}_{\mu}^{T}\right) n^{\mu} \leq 0,
\end{gathered}
$$

where, not only the choice of $X$ but also the coupling constants $\alpha, \beta$ are 'microlocalised'. To put it another way, using the linearity of the currents, we seek a microlocalised version of the combined currents:

$$
\mathbf{J}_{\mu}^{X+\beta N+\alpha T, w} \text {. }
$$

In our formalism (see Section 8.4), what we are seeking, for each frequency triple $(\omega, m, \ell)$, a combination $Q$ of $Q_{T}, Q_{\text {red }}^{z}$ and the virial currents $Q_{0}, Q_{1}, Q_{2}$ such that (57) holds and moreover

$$
Q[\infty]-Q[-\infty] \geq 0
$$

so we may drop the boundary terms and write

$$
b \int_{R_{0}^{*}}^{R_{1}^{*}}\left|u^{\prime}\right|^{2}+\chi\left(\lambda+\omega^{2}\right)|u|^{2}+|u|^{2} \leq\left|\int_{-\infty}^{\infty} H \cdot(f, h, y) \cdot\left(u, u^{\prime}\right)\right| .
$$

9.3.3. Summing and completing the argument. If we succeed in obtaining (61) for all frequency triples, then as in Section 9.2.3, we may sum, where now we obtain simply:

$$
b \int_{0}^{\infty} d \tau \int_{\Sigma_{\tau} \cap\left\{r \in\left[R_{0}, R_{1}\right]\right\}}\left(\chi|\partial \psi|^{2}+\left|\partial_{r^{*}} \psi\right|^{2}+|\psi|^{2}\right) \leq \int_{\Sigma_{0}}|\partial \psi|^{2} .
$$

The difficulty of the $\Sigma_{\tau_{f}}$ integral does not occur now in view of the new definition of the cutoffs. The rest of the proof follows as in Section 9.2.4, completing the argument for closedness.

We have thus reduced closedness to showing (61) for all frequency ranges. We shall show this in Section 11, completing thus the proof of Theorem 1. 


\section{The SMAll $a$ CASE}

This section and the next will contain the microlocalised estimates appealed to in the proof of Theorem 1 as given in Section 9. We begin by partitioning the frequency space into relevant frequency ranges.

10.1. The frequency ranges. Let $\omega_{1}, \lambda_{1}$ be (potentially large) parameters to be determined, and $\lambda_{2}$ be a (potentially small) parameter to be determined. We define the frequency ranges $\mathcal{F}_{b}, \mathcal{F}_{b}, \mathcal{F}_{\mathfrak{h}}, \mathcal{F}_{\sharp}$ by

- $\mathcal{F}_{b}=\left\{(\omega, m, \ell):|\omega| \leq \omega_{1}, \lambda_{m \ell}(a \omega) \leq \lambda_{1}\right\}$

- $\mathcal{F}_{\hbar}=\left\{(\omega, m, \ell):|\omega| \leq \omega_{1}, \lambda_{m \ell}(a \omega)>\lambda_{1}\right\}$

- $\mathcal{F}_{\mathfrak{b}}=\left\{(\omega, m, \ell):|\omega| \geq \omega_{1}, \lambda_{m \ell}(a \omega) \geq \lambda_{2} \omega^{2}\right\}$

- $\mathcal{F}_{\sharp}=\left\{(\omega, m, \ell):|\omega| \geq \omega_{1}, \lambda_{m \ell}(a \omega)<\lambda_{2} \omega^{2}\right\}$.

Our constructions will have a separate philosophy for each of the above ranges.

10.2. The $\mathcal{F}_{b}$ range (bounded frequencies). This is a compact frequency range and, in view of the fact that we have already constructed multipliers in the Schwarzschild case (see $[14,16]$ ), by stability considerations, their positivity properties carry over for $|a| \ll M$. See our proof in [19].

In [18], we chose to give a different argument, which, on one hand, allowed us to treat the general axi-symmetric case $(|a|<M, m=0)$, and furthermore, is more useful in applications to the openness regime of the general case.

Let us define

$$
V_{\text {new }}=\frac{4 M r a m \omega-a^{2} m^{2}}{\left(r^{2}+a^{2}\right)^{2}},
$$

$$
\begin{aligned}
V_{+} & =V-V_{\text {new }} \\
& =\frac{\Delta}{\left(r^{2}+a^{2}\right)^{4}}\left(\left(\lambda_{m \ell}+\omega^{2} a^{2}\right)\left(r^{2}+a^{2}\right)^{2}+\left(2 M r^{3}+a^{2} r^{2}+a^{4}-4 M r a^{2}\right)\right) .
\end{aligned}
$$

We have

$$
V_{+}>0
$$

for $r>r_{+}$. Moreover, according to our conventions, for all $|a| \leq a_{s} \ll M$,

$$
B\left(\Delta / r^{2}\right) \Lambda r^{-2}+B r^{-3} \geq V_{+} \geq\left(\Delta / r^{2}\right) b \Lambda r^{-2}+b\left(\Delta / r^{2}\right) r^{-3} .
$$

We may now fix an arbitrary $r_{+}+s_{-}>r_{c}>2 M$, where $s_{-}$is as in (33) and can be chosen only to depend on $a_{s}$, and it follows that for sufficiently small $a_{s}$ depending in particular on this choice, we have for $|a| \leq a_{s}$ and $(\omega, m, \ell) \in \mathcal{F}_{b}$ the following inequality in the region $r \geq r_{c}$ :

$$
B\left(\Delta / r^{2}\right) \Lambda r^{-2}+B r^{-3} \geq V \geq\left(\Delta / r^{2}\right) b \Lambda r^{-2}+b\left(\Delta / r^{2}\right) r^{-3},
$$

whereas, for all $r>r_{+}$, we have

$$
\left|V^{\prime}\left(r^{*}\right)\right| \leq B\left(\Delta / r^{2}\right) \Lambda r^{-3}+B\left(\Delta / r^{2}\right) r^{-4} .
$$

Finally, again choosing $a_{s}$ sufficiently small, it follows that for all $r \leq r_{c}$

$$
V^{\prime}\left(r^{*}\right) \geq b\left(\Delta / r^{2}\right) \Lambda
$$

whereas there exists a constant $R_{c}$ such that for $r \geq R_{c}$,

$$
-V^{\prime}\left(r^{*}\right) \geq\left(\Delta / r^{2}\right) b \Lambda r^{-3}+b\left(\Delta / r^{2}\right) r^{-4}
$$

in this frequency range. 
We will now split the frequency range $\mathcal{F}_{b}$ into two subcases, considering each separately.

10.2.1. The subrange $|\omega| \leq \omega_{3}$ (the near-stationary subcase). The motivation for the current to be constructed here is that in the Schwarzschild or $m=0$ case, applying $Q_{1}^{h}$ with $h=1$ immediately excludes nontrivial stationary solutions $\omega=0$.

We will fix an $\omega_{3}>0$ which will be constrained in this subsection to be small. Because $\omega_{3}$ is not exactly 0 the naive current $h=1$ must be modified.

We begin with a $Q_{1}^{h}$ current which will be defined with an $h=h\left(r^{*}\right)$.

Note first that given arbitrary $a_{0}<M$ and $q>0, p>0, R_{c}>0$ such that $e^{-p^{-1}} R_{c}$ is sufficiently large and $p$ sufficiently small, for each $|a| \leq a_{s}$, we can define a function $h\left(r^{*}\right)$, such that the following hold: For $r \leq r_{c}$,

$$
0 \leq h \leq R_{c}^{-2}, \quad\left|h^{\prime \prime}\left(r^{*}\right)\right| \leq q /\left|r^{*}\right|^{2},
$$

with $h\left(r^{*}\right)$ moreover of compact support when restricted to $-\infty<r^{*} \leq r_{c}^{*}$, whereas for $r_{c} \leq r \leq e^{-p^{-1}} R_{c}$,

$$
h \geq R_{c}^{-2} \Delta / r^{2}, \quad h^{\prime \prime}\left(r^{*}\right) \leq 0,
$$

whereas for $e^{-p^{-1}} R_{c} \leq r \leq R_{c}$,

$$
\left|h^{\prime}\left(r^{*}\right)\right| \leq 4 R_{c}^{-2} p / r, \quad\left|h^{\prime \prime}\left(r^{*}\right)\right| \leq 4 R_{c}^{-2} p / r^{2},
$$

whereas, finally, for $r \geq R_{c}$,

$$
h=0 .
$$

This $h$ will be useful in view of the positivity of $V_{+}$. We note finally that $h$ can be chosen so that $h$ restricted to $r \geq r_{c}$ is independent of the parameter $q$.

Let us also consider a current $Q_{2}^{y}$ defined with a $y=y\left(r^{*}\right)$. Like $h$, the function $y$ will be independent of $\omega, m$, and $\ell$ in the allowed range. Given the parameters $R_{c}$, $p$, with $e^{-p^{-1}} R_{3}$ sufficiently large and $p$ sufficiently small, the function will satisfy the following properties: We set $y\left(r_{c}^{*}\right)=0$, and for $r \leq r_{c}$,

$$
y^{\prime}\left(r^{*}\right)=h
$$

noting that $y$ is bounded below in $r \leq r_{c}$ by a potentially large negative constant depending on $q$, in view of the fact that $h$ is identically 0 for sufficiently low $r^{*}$. For $r_{c} \leq r \leq e^{-p^{-1}} R_{c}$, we require

$$
y^{\prime}\left(r^{*}\right) \geq 0, \quad(y V)^{\prime}\left(r^{*}\right) \leq \frac{1}{2} R_{c}^{-2} \Delta / r^{2},
$$

whereas for $e^{-p^{-1}} R_{c} \leq r \leq R_{c}$

$$
y^{\prime}\left(r^{*}\right) \geq b R_{c}^{-2}, \quad-(y V)^{\prime}\left(r^{*}\right) \geq b R_{c}^{-2} / r^{2},
$$

whereas finally, for $r \geq R_{c}$,

$$
y=1 .
$$

In general, for sufficiently large $e^{-p^{-1}} R_{c}$ and small $p$, we can indeed construct such a $y$ in view essentially of (64), (65) and (67). Let us add that $y$ restricted to $r \geq r_{c}$ can be chosen independently of the choice of $h$.

Consider now the current $Q=Q_{1}^{h}+Q_{2}^{y}$. In $r \leq r_{c}$, recall the one-sided bound

$$
-y V^{\prime} \geq 0
$$

which follows from (66). It follows that in $r \leq r_{c}$,

$$
Q^{\prime}\left(r^{*}\right) \geq-q\left|r^{*}\right|^{-2}|u|^{2}+h \operatorname{Re}(u \bar{H})+2 y \operatorname{Re}\left(u^{\prime} \bar{H}\right) .
$$


For $r_{c} \leq r \leq e^{-p^{-1}} R_{c}$, choosing $p$ appropriately, choosing $\omega_{3}, a_{s}$ sufficiently small

$$
Q^{\prime}\left(r^{*}\right) \geq R_{c}^{-2}\left(\Delta / r^{2}\right)\left|u^{\prime}\right|^{2}+b V_{+}|u|^{2}+h \operatorname{Re}(u \bar{H})+2 y \operatorname{Re}\left(u^{\prime} \bar{H}\right) .
$$

For $e^{-p^{-1}} R_{c} \leq r \leq R_{c}$, we have for $p, \omega_{3}$ suitably small

$$
Q^{\prime}\left(r^{*}\right) \geq b R_{c}^{-2}\left(\Delta / r^{2}\right)\left|u^{\prime}\right|^{2}+h \operatorname{Re}(u \bar{H})+2 y \operatorname{Re}\left(u^{\prime} \bar{H}\right) .
$$

Finally, for $r \geq R_{c}$ we have,

$$
Q^{\prime}\left(r^{*}\right)=\left(Q_{2}^{y}\right)^{\prime}\left(r^{*}\right) \geq 2 y \operatorname{Re}\left(u^{\prime} \bar{H}\right) .
$$

We obtain finally,

$$
\begin{aligned}
b\left(\lambda_{1}\right) \int_{r_{c}^{*}}^{R_{c}^{*}} & \left(\Delta / r^{2}\right)\left|u^{\prime}\right|^{2}+\left(\Delta / r^{2}\right) r^{-3}\left(1+\Lambda+\omega^{2}\right)|u|^{2} d r^{*} \\
\leq & \int_{-\infty}^{r_{c}^{*}} q\left|r^{*}\right|^{-2}|u|^{2} d r^{*}+\int_{-\infty}^{+\infty}\left(2 y \operatorname{Re}\left(u^{\prime} \bar{H}\right)+h \operatorname{Re}(\bar{H} u)\right) d r^{*} \\
& +Q_{2}^{y}(\infty)-\left(Q_{1}^{h}+Q_{2}^{y}\right)(-\infty) .
\end{aligned}
$$

The above is the prototype of the type of inequality we derive for all frequencies. Cf. (58). Note, however, the extra term proportional to $q$. We shall deal with this in Section 10.6.

10.2.2. The subrange $|\omega| \geq \omega_{3}$ (the non-stationary subcase). The construction of this section will yield a positive current for $\omega_{3}$ small and $\omega_{1}, \lambda_{1}$ large depending on the smallness of $a_{s}$.

Let us note that in fact, the construction of this section is quite general and depends only on the (almost) positivity properties of the potential $V$ together with its asymptotic properties, and not, in particular, on the sign of $V^{\prime}$, which will be crucial in Section 10.4. We note that similar constructions have a long tradition in spectral theory and are typically used to prove continuity of the spectrum away from the $\omega=0$.

We consider a $Q_{2}^{y}$-current. Recall that we have

$$
\begin{aligned}
\left(Q_{2}^{y}\right)^{\prime}\left(r^{*}\right) & =y^{\prime}\left|u^{\prime}\right|^{2}+\omega^{2} y^{\prime}|u|^{2}-(y V)^{\prime}|u|^{2}+y \operatorname{Re}\left(u^{\prime} \bar{H}\right) \\
& =y^{\prime}\left|u^{\prime}\right|^{2}+\omega^{2} y^{\prime}|u|^{2}-\left(y V_{+}\right)^{\prime}|u|^{2}-y^{\prime} V_{\text {new }}|u|^{2}-y V_{\text {new }}^{\prime}|u|^{2}+y \operatorname{Re}\left(u^{\prime} \bar{H}\right) .
\end{aligned}
$$

Note that for general bounded $y \geq 0, y^{\prime}>0$, we have

$$
\begin{aligned}
\int_{-\infty}^{\infty}\left(y V_{+}\right)^{\prime}|u|^{2}= & -\int_{-\infty}^{\infty} y V_{+}\left(u \bar{u}^{\prime}+u^{\prime} \bar{u}\right)+y V_{+}|u|^{2}(\infty)-y V_{+}|u|^{2}(-\infty) \\
\leq & \frac{1}{2} \int_{-\infty}^{\infty} y^{\prime}\left|u^{\prime}\right|^{2}+2 \int_{-\infty}^{\infty}\left(y^{2} V_{+}^{2} / y^{\prime}\right)|u|^{2} \\
& +y V_{+}|u|^{2}(\infty)-y V_{+}|u|^{2}(-\infty) \\
\leq & \frac{1}{2} \int_{-\infty}^{\infty} y^{\prime}\left|u^{\prime}\right|^{2}+2 \int_{-\infty}^{\infty}\left(y^{2} V_{+}^{2} / y^{\prime}\right)|u|^{2}
\end{aligned}
$$

We now define

$$
y=e^{-2 \omega_{3}^{-1} \int_{r^{*}}^{\infty} V_{+} d r^{*}}
$$

Note that in this case, we have that

$$
y^{\prime}=2 \omega_{3}^{-1} V_{+} e^{-2 \omega_{3}^{-1} \int_{r^{*}}^{\infty} V_{+} d r^{*}} .
$$


We thus have for this choice of $y$ that

$$
\int_{-\infty}^{\infty}\left(y V_{+}\right)^{\prime}|u|^{2} \leq \frac{1}{2} \int_{-\infty}^{\infty} y^{\prime}\left|u^{\prime}\right|^{2}+\frac{1}{2} \int_{-\infty}^{\infty} \omega_{3}^{2} y^{\prime}|u|^{2} .
$$

The potential $V_{\text {new }}$ obeys the estimates

$$
\left|V_{\text {new }}\right| \leq a_{s} B\left(\lambda_{1}+a_{s}^{2} \omega_{1}^{2}\right) r^{-4}, \quad\left|V_{\text {new }}^{\prime}\right| \leq a_{s} B \Delta / r^{2}\left(\lambda_{1}+a_{s}^{2} \omega_{1}^{2}\right) r^{-5} .
$$

Therefore, the term $\frac{1}{2} \omega_{3}^{2} y^{\prime}|u|^{2}$ dominates both $-y^{\prime} V_{\text {new }}|u|^{2}$ and $-y V_{\text {new }}^{\prime}|u|^{2}$ in (70), provided that $a_{s} \ll \omega_{3}^{2}$. Integrating, we finally obtain the inequality:

$$
\begin{array}{r}
b\left(\omega_{3}, \omega_{1}, \lambda_{1}\right) \int_{r_{c}^{*}}^{R_{c}^{*}}\left(\Delta / r^{2}\right)\left|u^{\prime}\right|^{2}+\left(\Delta / r^{2}\right)\left(1+\left(\Lambda+\omega^{2}\right)|u|^{2}\right. \\
\leq \int_{-\infty}^{\infty} 2 y \operatorname{Re}\left(u^{\prime} \bar{H}\right)+Q_{2}^{y}(\infty)-Q_{2}^{y}(-\infty) .
\end{array}
$$

10.3. The $\mathcal{F}_{\hbar}$ range (angular dominated frequencies). The considerations of this section will constrain $\lambda_{1}$ to be suitably large, depending on the choice of $\omega_{1}$. This range is in fact covered by the arguments in Section 11 for the general case $|a|<M$, and, of course, in [18]. Briefly, a simple construction in this regime involves the current $Q=Q_{0}^{f}+Q_{1}^{h}$ with a positive and monotonically increasing $f$ and a function $h$ of compact support peaking in the region where $V$ dominates $\omega^{2}$. The final resulting estimate is:

$$
\begin{aligned}
b \int_{r_{c}^{*}}^{R_{c}^{*}} & \left(\left|u^{\prime}\right|^{2}+\left(\Delta / r^{2}\right) r^{-3}\left(1+\left(\Lambda+\omega^{2}\right)|u|^{2}\right)\right. \\
\leq & \int_{-\infty}^{\infty} 2 f \operatorname{Re}\left(u^{\prime} \bar{H}\right)+\left(f^{\prime}+h\right) \operatorname{Re}(\bar{H} u) \\
& +Q_{0}^{f}(\infty)-Q_{0}^{f}(-\infty) .
\end{aligned}
$$

10.4. The $\mathcal{F}_{h}$ range (trapped frequencies). This is the frequency range of trapping. It is once again subsumed by the trapped case $\mathcal{G}_{\natural}$ which is treated in detail in Section 11.

Thus, we take the opportunity here to merely highlight the simpler construction in [18] which is special to the $|a| \ll M$ case. This is a good warm-up for following the detailed but more technical construction of $\mathcal{G}_{\mathrm{h}}$ in Section 11.5.

The main observation is that $V^{\prime}$ has a unique non-degenerate zero $r_{m \ell}^{(a \omega)}$ for $(\omega, m, \ell) \in \mathcal{F}_{\mathfrak{h}}$, depending smoothly on $\omega$. This can be thought of as a quantised version of the property of trapped null orbits described at the end of Section 3.2.

We consider the current $Q_{0}^{f}$ :

$$
\left(Q_{0}^{f}\right)^{\prime}=2 f^{\prime}\left|u^{\prime}\right|^{2}-f V^{\prime}|u|^{2}-\frac{1}{2} f^{\prime \prime \prime}|u|^{2}+2 f \operatorname{Re}\left(u^{\prime} \bar{H}\right)+f^{\prime} \operatorname{Re}(u \bar{H}) .
$$

The function $f$ is chosen in such a way that

(1) $f^{\prime}\left(r^{*}\right) \geq 0$,

(2) $f<0$ for $r<r_{m \ell}^{(a \omega)}$ and $f>0$ for $r>r_{m \ell}^{(a \omega)}$,

(3) $f^{\prime \prime \prime}<0$ in the neighborhood of $r_{m \ell}^{(a \omega)}$,

(4) $-f V^{\prime}>0$ dominates outside of a small neighborhood $r_{m \ell}^{(a \omega)}$. 
We obtain finally

$$
\begin{gathered}
b \int_{r_{c}^{*}}^{R_{c}^{*}}\left(\left(\Delta / r^{2}\right) r^{-2}\left|u^{\prime}\right|^{2}+\left(\Delta / r^{2}\right) r^{-2}|u|^{2}+\Delta / r^{2} r^{-5}\left(r-r_{m \ell}^{(a \omega)}\right)^{2}\left(\Lambda+\omega^{2}\right)|u|^{2}\right) \\
\leq \int_{-\infty}^{\infty} 2 f \operatorname{Re}\left(u^{\prime} \bar{H}\right)+f^{\prime} \operatorname{Re}(\bar{H} u)+Q_{0}^{f}(\infty)-Q_{0}^{f}(-\infty) .
\end{gathered}
$$

10.5. The $\mathcal{F}_{\sharp}$ range (time-dominated frequencies). Again, this range is subsumed by the $\mathcal{G}_{\sharp}$ regime in the general $|a|<M$ treated in detail in Section 11, so here we can be brief.

For small enough $\lambda_{2}$ and large enough $\omega_{1}$, we have

$$
\omega^{2}-V \geq \frac{1-c}{2} \omega^{2}
$$

for frequencies in $\mathcal{F}_{\sharp}$. We apply the current $Q_{2}^{y}$ with a function $y$ chosen so that $y \geq 0, y^{\prime} \geq B\left(\Delta / r^{2}\right) r^{-2}$ in $\left(r_{+}, R_{c}\right]$ and $y=1$ for $y \geq R_{c}+1$. Since, for $(\omega, m, \ell) \in \mathcal{F}_{\sharp}$, we have

$$
\left|V^{\prime}\right| \leq B\left(\Delta / r^{2}\right) r^{-3}\left(\left(\lambda_{m \ell}+a_{0}^{2} \omega^{2}\right)+1\right) .
$$

We obtain

$$
\begin{aligned}
& b \int_{r_{c}^{*}}^{R_{c}^{*}} \Delta / r^{2} r^{-2}\left(\left|u^{\prime}\right|^{2}+\left(\omega^{2}+\Lambda\right)|u|^{2}\right) \\
& \quad \leq \int_{-\infty}^{\infty} 2 y \operatorname{Re}\left(u^{\prime} \bar{H}\right)+Q_{2}^{y}(\infty)-Q_{2}^{y}(-\infty) .
\end{aligned}
$$

See Section 11 (or alternatively [18]) for more details.

10.6. A note on summation and the red-shift. We have obtained inequalities (58) for all frequency triples, except for an extra term

$$
\int_{-\infty}^{r_{c}^{*}} q\left|r^{*}\right|^{-2}|u|^{2} d r^{*}
$$

coming from (68) corresponding to the $|\omega| \leq \omega_{3}$ sub-case of the $\mathcal{F}_{b}$ frequency range.

After summing, we thus in fact obtain:

$$
\begin{aligned}
b \int_{0}^{\infty} d \tau \int_{\Sigma_{\tau} \cap\left\{r \in\left[R_{0}, R_{1}\right]\right\}}\left(\chi|\partial \psi|^{2}+|\psi|^{2}\right) & \leq \int_{\Sigma_{0}}|\partial \psi|^{2}+q \int_{0}^{\infty} d \tau \int_{\Sigma_{\tau} \cap\left\{r \leq r_{c}\right\}}|\partial \psi|^{2} \\
& +\int_{\mathcal{H}^{+}}\left|\mathbf{J}_{\mu}^{T} n_{\mathcal{H}}^{\mu}\right|+\int_{\mathcal{I}^{+}} \mathbf{J}_{\mu}^{T} n_{\mathcal{I}}^{\mu}
\end{aligned}
$$

We now add the red-shift estimate (33) and the $\mathbf{J}^{T}$ identity (47) to eliminate the small $q$-term and the $\mathcal{H}^{+}, \mathcal{I}^{+}$contributions. We obtain again (cf. (60))

$$
b \int_{0}^{\infty} d \tau \int_{\Sigma_{\tau} \cap\left\{r \in\left[R_{0}, R_{1}\right]\right\}}\left(\chi|\partial \psi|^{2}+|\psi|^{2}\right) \leq \int_{\Sigma_{0}}|\partial \psi|^{2} .
$$

This is the desired integrated local energy decay estimate, which may now be coupled with the red-shift estimate and the estimates at null infinity (41) of section 5 to obtain the statements of decay for the non-degenerate energy and pointwise decay for the solution of Theorem 1 . 


\section{The general $|a|<M$ CASE}

In Section 9.3.3, we have reduced the problem to obtaining the inequality (61) for all frequency ranges. In this section, we shall obtain precisely these inequalities, completing thus the proof.

We begin with a closer examination of some properties of the potential $V=$ $V_{m \ell}^{(a \omega)}(r)$. Without loss of generality we may assume that the specific angular momentum $a$ is positive $0<a<M$, as the problem admits a symmetry with respect to simultaneous reversal of the signs of $a$ and the azimuthal frequency $m$.

11.1. Properties of the potential $V$. We decompose the potential $V=V_{0}+V_{1}$ with

$$
\begin{aligned}
& V_{0}=\frac{4 M r a m \omega-a^{2} m^{2}+\Delta\left(\lambda_{m \ell}+\omega^{2} a^{2}\right)}{\left(r^{2}+a^{2}\right)^{2}}, \\
& V_{1}=\frac{\Delta\left(3 r^{2}-4 M r+a^{2}\right)}{\left(r^{2}+a^{2}\right)^{3}}-\frac{3 \Delta^{2} r^{2}}{\left(r^{2}+a^{2}\right)^{4}} .
\end{aligned}
$$

We note that $V_{1}$ does not contain any frequency parameters and that

$$
V_{1}=\frac{\Delta}{\left(r^{2}+a^{2}\right)^{4}}\left[a^{2} \Delta+2 M r\left(r^{2}-a^{2}\right)\right] \geq 0
$$

Lemma 11.1.1. For any values of the parameters $\Lambda>0, m$ and $\omega$ the potential function $V_{0}$ on the interval $\left(r_{+}, \infty\right)$ has at most one maximum and one minimum, achieved at the respective points $r_{\max }^{0}$ and $r_{\min }^{0}$ with the property that $r_{\min }^{0}<r_{\max }^{0}$. Moreover, for all sufficiently large $\Lambda$ the value $r_{\text {max }}^{0}$ is bounded uniformly from above provided that either $m \omega \geq 0$ or $a^{2} \omega^{2} \leq C \Lambda$ for some constant $C$, in which case the bound for $r_{\max }^{0}$ may depend on $C$.

Proof. We compute

$$
\begin{aligned}
\frac{d}{d r} V_{0} & =4 m a M \omega\left[\frac{1}{\left(r^{2}+a^{2}\right)^{2}}-\frac{4 r^{2}}{\left(r^{2}+a^{2}\right)^{3}}\right]+\frac{4 r a^{2} m^{2}}{\left(r^{2}+a^{2}\right)^{3}}+\frac{\Lambda}{\left(r^{2}+a^{2}\right)^{2}}\left[2(r-M)-\frac{4 r \Delta}{r^{2}+a^{2}}\right] \\
& =\frac{1}{\left(r^{2}+a^{2}\right)^{3}}\left[4 m a M \omega\left(-3 r^{2}+a^{2}\right)+4 r a^{2} m^{2}\right] \\
& +\frac{\Lambda}{\left(r^{2}+a^{2}\right)^{3}}\left(2 r^{3}+2 a^{2} r-2 M r^{2}-2 M a^{2}-4 r^{3}+8 M r^{2}-4 r a^{2}\right) \\
& =\frac{1}{\left(r^{2}+a^{2}\right)^{3}}\left[4 m a M \omega\left(-3 r^{2}+a^{2}\right)+4 r a^{2} m^{2}-2 \Lambda\left(r^{3}+a^{2} r-3 M r^{2}+M a^{2}\right)\right] .
\end{aligned}
$$

Thus, we have

$$
\begin{aligned}
\frac{d}{d r}\left[\left(r^{2}+a^{2}\right)^{3} \frac{d}{d r} V_{0}\right] & =-24 M a m \omega r+4 a^{2} m^{2}-2 \Lambda\left(3 r^{2}-6 M r+a^{2}\right) \\
& =-6 \Lambda\left[r^{2}-2 M r+4 M r \sigma+\frac{a^{2}}{3}-\frac{2}{3} a^{2} \frac{m^{2}}{\Lambda}\right]
\end{aligned}
$$

where we set

$$
\sigma=\frac{a m \omega}{\Lambda} \text {. }
$$

We now examine the points where $\frac{d}{d r}\left[\left(r^{2}+a^{2}\right)^{3} \frac{d}{d r} V_{0}\right]$ vanishes. Such points are solutions of the equation

$$
r^{2}-2 M r(1-2 \sigma)+\frac{a^{2}}{3}\left(1-\frac{2 m^{2}}{\Lambda}\right)=0,
$$


let us denote them by:

$$
r_{1,2}=M(1-2 \sigma) \pm \sqrt{M^{2}(1-2 \sigma)^{2}-\frac{a^{2}}{3}\left(1-\frac{2 m^{2}}{\Lambda}\right)} .
$$

We recall the horizon is determined by the value $r_{+}: r_{+}^{2}-2 M r_{+}+a^{2}=0$. In particular, $r_{+}>M$ for all values of $a<M$.

If $\sigma>0$ then $r_{2}<M$ and the only point where $\frac{d}{d r}\left[\left(r^{2}+a^{2}\right)^{3} \frac{d}{d r} V_{0}\right]=0$ on the interval $\left(r_{+}, \infty\right)$ is

$$
r_{1}=M(1-2 \sigma)+\sqrt{M^{2}(1-2 \sigma)^{2}-\frac{a^{2}}{3}\left(1-\frac{2 m^{2}}{\Lambda}\right)} .
$$

In that case we note that the function $\left(r^{2}+a^{2}\right)^{3} \frac{d}{d r} V_{0} \rightarrow-\infty$ as $r \rightarrow \infty$, as long as $\Lambda>0$. Therefore, $\frac{d}{d r} V_{0}$ can vanish at most at two points $r_{\text {min }}^{0}$ and $r_{\text {max }}^{0}$, where

$$
\frac{d^{2}}{d r^{2}} V_{0}\left(r_{\min }^{0}\right)>0, \quad \frac{d^{2}}{d r^{2}} V_{0}\left(r_{\max }^{0}\right)<0
$$

and $r_{\text {min }}^{0}<r_{\text {max }}^{0}$.

If $r_{1}$ is not real then $\frac{d}{d r}\left[\left(r^{2}+a^{2}\right)^{3} \frac{d}{d r} V_{0}\right]$ remains negative for all $r \geq r_{+}$, which means that $\frac{d}{d r} V_{0}$ can only vanish at one point, where $V_{0}$ has a maximum.

If $\sigma<0$ then we must examine

$r_{2}=M(1-2 \sigma)-\sqrt{M^{2}(1-2 \sigma)^{2}-\frac{a^{2}}{3}\left(1-\frac{2 m^{2}}{\Lambda}\right)}=M(1-2 \sigma)\left[1-\sqrt{1-\frac{a^{2}\left(1-\frac{2 m^{2}}{\Lambda}\right)}{3 M^{2}(1-2 \sigma)^{2}}}\right]$.

Since $a<M$ and $\sigma<0$, we have

$$
\frac{a^{2}\left(1-\frac{2 m^{2}}{\Lambda}\right)}{3 M^{2}(1-2 \sigma)^{2}}<\frac{1}{3}
$$

We note that for $0 \leq x<\frac{1}{3}$,

$$
\sqrt{1-x}>1-\frac{2 x}{3}
$$

which implies that

$$
r_{2}<\frac{2 M(1-2 \sigma) a^{2}\left(1-\frac{2 m^{2}}{\Lambda}\right)}{9 M^{2}(1-2 \sigma)^{2}}=\frac{2 a^{2}\left(1-\frac{2 m^{2}}{\Lambda}\right)}{9 M(1-2 \sigma)}<\frac{2}{9} M .
$$

This now implies that $r_{1}$ is the only zero of $\frac{d}{d r}\left[\left(r^{2}+a^{2}\right)^{3} \frac{d}{d r} V_{0}\right]$ on the interval $\left[r_{+}, \infty\right)$ and the previous argument applies.

The last statement of the lemma easily follows from observing that for all sufficiently large $\Lambda$ the leading order behavior of $\left[\left(r^{2}+a^{2}\right)^{3} \frac{d}{d r} V_{0}\right]$ as $r \rightarrow \infty$ is determined by the expression

$$
(6 \Lambda M-12 M a m \omega) r^{2}-2 \Lambda r^{3} .
$$

The next statement effectively establishes that even if $r_{m i n}$ exists, it can only be 'trapped' for the value $\omega=\omega_{+}$. 
Lemma 11.1.2. For all values of the parameters $\Lambda, \omega, m$, we have that on the horizon

$$
\omega^{2} \geq V\left(r_{+}\right)
$$

with equality achieved only for $\omega=\omega_{+}$. In particular, in the notations of the previous lemma, this implies that

$$
\omega^{2}>V_{0}\left(r_{\min }\right) \text {. }
$$

Proof. We compute

$$
\begin{aligned}
\omega^{2}-V\left(r_{+}\right) & =\omega^{2}-\frac{4 M r_{+} a m \omega-a^{2} m^{2}}{\left(r_{+}^{2}+a^{2}\right)^{2}} \\
& =\frac{1}{4 M^{2} r_{+}^{2}}\left[4 M^{2} r_{+}^{2} \omega-4 M r_{+} a m \omega+a^{2} m^{2}\right]=\frac{\left(2 M r_{+} \omega-a m\right)^{2}}{4 M^{2} r_{+}^{2}} .
\end{aligned}
$$

The equality

$$
\omega^{2}=V\left(r_{+}\right)
$$

occurs precisely at the threshold of the super-radiance condition

$$
\omega=\omega_{+}=\frac{a m}{2 M r_{+}} \text {. }
$$

We now argue that in the super-radiant regime $V_{0}$ has only a maximum, that is the point $r_{\text {min }}$ is absent.

Lemma 11.1.3. Let

$$
m \omega \leq \frac{a m^{2}}{2 M r_{+}} .
$$

Then

$$
\frac{d}{d r} V\left(r_{+}\right) \geq \frac{d}{d r} V_{0}\left(r_{+}\right) \geq c \Lambda>0 .
$$

Since we have already shown that $r_{\min }^{0}$, if exists, has the property that $r_{\min }^{0}<r_{\max }^{0}$ this implies that $r_{\text {min }}^{0}$ does not exist and the potential $V_{0}$ has its unique critical point at $r_{\max }^{0}$.

Remark 11.1. The conclusion of the lemma can be easily extended to the range

$$
a m \omega \leq \frac{a^{2} m^{2}}{2 M r_{+}}+\alpha \Lambda
$$

for a sufficiently small constant $\alpha$. The smallness of $\alpha$ depends on the smallness of the quantity $(M-a)$.

Proof. We compute

$$
\begin{aligned}
\frac{d}{d r} V_{0}\left(r_{+}\right) & =\frac{4 m a M \omega}{\left(r_{+}^{2}+a^{2}\right)^{3}}\left[-3 r_{+}^{2}+a^{2}\right]+\frac{4 r_{+} a^{2} m^{2}}{\left(r_{+}^{2}+a^{2}\right)^{3}}+\frac{2\left(r_{+}-M\right) \Lambda}{\left(r_{+}^{2}+a^{2}\right)^{2}} \\
& =\frac{1}{\left(r_{+}^{2}+a^{2}\right)^{3}}\left[4 m a M \omega\left[-3 r_{+}^{2}+a^{2}\right]+4 r_{+} a^{2} m^{2}+2\left(r_{+}^{2}+a^{2}\right)\left(r_{+}-M\right) \Lambda\right]
\end{aligned}
$$

For the values of the parameters $m \omega<0$ the conclusion of the lemma is obvious. Otherwise, using the condition

$$
m \omega \leq \frac{a m^{2}}{2 M r_{+}}
$$


we obtain

$$
\begin{aligned}
\left(r_{+}^{2}+a^{2}\right)^{3} \frac{d}{d r} V_{0}\left(r_{+}\right) & \geq\left[\frac{2 a^{2} m^{2}}{r_{+}}\left(-3 r_{+}^{2}+a^{2}\right)+4 r_{+} a^{2} m^{2}+2\left(r_{+}^{2}+a^{2}\right)\left(r_{+}-M\right) \Lambda\right] \\
& =\left[\frac{2 a^{2} m^{2}}{r_{+}}\left(-r_{+}^{2}+a^{2}\right)+2\left(r_{+}^{2}+a^{2}\right)\left(r_{+}-M\right) \Lambda\right] \\
& =2\left(r_{+}-M\right)\left[\Lambda\left(r_{+}^{2}+a^{2}\right)-2 a^{2} m^{2}\right] \\
& =4\left(r_{+}-M\right)\left[\Lambda M r_{+}-a^{2} m^{2}\right] .
\end{aligned}
$$

The inequalities $\Lambda \geq m^{2}$ and $r_{+}>M>a$ imply that $\frac{d}{d r} V_{0}\left(r_{+}\right)>0$. We finish the proof by observing that

$$
\frac{d}{d r} V_{1}\left(r_{+}\right)=\frac{4 M r_{+}\left(r_{+}-M\right)\left(r_{+}^{2}-a^{2}\right)}{\left(r_{+}^{2}+a^{2}\right)^{4}}>0 .
$$

Our final result of this section gives a mathematical embodiment to the miracle which is key to the validity of Theorem 1, namely, the fact that superradiant frequencies are not 'trapped'.

Lemma 11.1.4. For the values $0 \leq a<M$ and

$$
0 \leq m \omega \leq \frac{a m^{2}}{2 M r_{+}} .
$$

we have

$$
\omega^{2}<V_{0}\left(r_{\max }^{0}\right)
$$

Proof. Let $r_{0} \geq r_{+}$be such that

$$
m \omega=\frac{a m^{2}}{2 M r_{0}}
$$

We compute

$$
\begin{aligned}
\omega^{2}-V_{0}\left(r_{0}\right) & =\omega^{2}-\frac{4 M r_{0} a m \omega-a^{2} m^{2}+\Delta \Lambda}{\left(r_{0}^{2}+a^{2}\right)^{2}} \\
& =\frac{1}{\left(r_{0}^{2}+a^{2}\right)^{2}}\left[\left(r_{0}^{2}+a^{2}\right)^{2} \omega^{2}-4 M r_{0} a m \omega+a^{2} m^{2}-\Delta \Lambda\right] \\
& =\frac{1}{\left(r_{0}^{2}+a^{2}\right)^{2}}\left[4 M^{2} r_{0}^{2} \omega^{2}-4 M r_{0} a m \omega+a^{2} m^{2}+\omega^{2}\left(\left(r_{0}^{2}+a^{2}\right)^{2}-4 M r_{0}^{2}\right)-\Delta \Lambda\right] \\
& =\frac{\omega^{2}\left(r_{0}^{2}-2 M r_{0}+a^{2}\right)\left(r_{0}^{2}+2 M r_{0}+a^{2}\right)-\Delta \Lambda}{\left(r_{0}^{2}+a^{2}\right)^{2}} \\
& =\frac{\Delta}{\left(r_{0}^{2}+a^{2}\right)^{2}}\left[\frac{a^{2} m^{2}}{4 M^{2}}\left(1+\frac{2 M}{r_{0}}+\frac{a^{2}}{r_{0}^{2}}\right)-\Lambda\right] .
\end{aligned}
$$

We now recall that $a<M<r_{0}$ and that $\Lambda \geq m(m+1)$ to conclude that

$$
\omega^{2}-V_{0}\left(r_{0}\right)<0 .
$$


11.2. The frequency ranges. Let $\omega_{1}, \lambda_{1}$ be (potentially large) parameters to be determined, and $\lambda_{2}$ be a (potentially small) parameter to be determined. We define the frequency ranges $\mathcal{G}_{b}, \mathcal{G}_{b}, \mathcal{G}_{\mathfrak{h}}, \mathcal{G}_{\sharp}, \mathcal{G} \sharp$ by

- $\mathcal{G}^{\sharp}=\left\{(\omega, m, \ell): \omega^{2}+\Lambda \geq \lambda_{1}, m \omega \in\left[0, \frac{a m^{2}}{2 M r_{+}}\right]\right\}$,

- $\mathcal{G}_{\sharp}=\left\{(\omega, m, \ell):|\omega| \geq \omega_{1}, \Lambda<\lambda_{2} \omega^{2}, m \omega \notin\left[0, \frac{a m^{2}}{2 M r_{+}}\right]\right\}$,

- $\mathcal{G}_{\hbar}=\left\{(\omega, m, \ell):|\omega| \geq \omega_{1}, \lambda_{2} \Lambda>\omega^{2}, m \omega \notin\left[0, \frac{a m^{2}}{2 M r_{+}}\right]\right\}$,

- $\mathcal{G}_{\text {h }}=\left\{(\omega, m, \ell):|\omega| \geq \omega_{1}, \lambda_{2} \Lambda \leq \omega^{2} \leq \lambda_{2}^{-1} \Lambda, m \omega \notin\left[0, \frac{a m^{2}}{2 M r_{+}}\right]\right\}$,

- $\mathcal{G}_{b}=\left\{(\omega, m, \ell):|\omega| \leq \omega_{1}, \Lambda \leq \lambda_{1}\right\}$.

11.3. The $\mathcal{G} \sharp$ range. This a large frequency super-radiant regime. The lower bound $\Lambda \geq m(m+1)$ together with the superradiant condition implies that in this regime

$$
\omega^{2} \leq\left(\frac{a}{2 M r_{+}}\right)^{2} \Lambda
$$

and therefore $\Lambda$ is necessarily large. In the superradiant range the conclusions of Lemma 11.1.3 and Lemma 11.1.4 apply. In particular, the potential $V_{0}$ has its only critical point-a maximum-at $r_{\max }^{0}$ and $\omega^{2}<V_{0}\left(r_{\max }^{0}\right)$. The latter in fact means that

$$
V_{0}(r)-\omega^{2} \geq c \Lambda, \quad \forall r \in\left[r_{\max }-\delta, r_{\max }+\delta\right]
$$

for some positive constant $c$ and $\delta>0$. Note that $\delta$ can be chosen to be universal and independent of the size of (large) $\Lambda$. We further observe that $r_{\max }^{0}$ is uniformly bounded and is away from the value of $r_{+}$:

$$
c \leq\left(r_{\max }-r_{+}\right) \leq C
$$

with the constants $c, C$ independent of $\Lambda$. We also have that the full potential $V=V_{0}+V_{1}$ has the property that

$$
\frac{d}{d r} V\left(r_{+}\right) \geq \frac{d}{d r} V_{0}\left(r_{+}\right) \geq c \Lambda .
$$

We now argue that the properties of $V$ mimic precisely those of $V_{0}$ with the value $r_{\max }^{0}$ replaced by $r_{\max }$ :

$$
\left|r_{\max }-r_{\max }^{0}\right| \leq c \Lambda^{-1} .
$$

In the regime under consideration, the second derivative

$$
\frac{d}{d r}\left(\left(r^{2}+a^{2}\right)^{3} \frac{d}{d r} V_{0}(r)\right)
$$

may only change its sign once at a point $r_{1}<r_{\max }^{0}$. If it happens then

$$
\frac{d}{d r} V_{0}(r) \geq c \Lambda
$$

perhaps with a slightly different value of $c$ for all $r \in\left[r_{+}, r_{1}\right]$ and, since

$$
\left|\frac{d}{d r}\left(\left(r^{2}+a^{2}\right)^{3} \frac{d}{d r} V_{0}(r)\right)\right| \leq C \Lambda,
$$

we have that

$$
\left(r_{\max }^{0}-r_{1}\right) \geq c .
$$

On the interval $\left[r_{1}, \infty\right)$, we have

$$
\frac{d}{d r}\left(\left(r^{2}+a^{2}\right)^{3} \frac{d}{d r} V_{0}(r)\right) \leq 0
$$


and we can choose a value $r_{1}^{\prime} \in\left(r_{1}, r_{\max }^{0}\right)$ such that

$$
\frac{d}{d r} V_{0}(r) \geq \frac{c}{2} \Lambda, \quad \forall r \in\left[r_{+}, r_{1}^{\prime}\right]
$$

and

$$
\frac{d}{d r}\left(\left(r^{2}+a^{2}\right)^{3} \frac{d}{d r} V_{0}(r)\right) \leq-c \Lambda r^{2}, \quad \forall r \in\left[r_{1}^{\prime}, \infty\right) .
$$

On the other hand, the potential $V_{1}$ obeys

$$
\left|V_{1}\right| \leq C r^{-3}, \quad\left|\frac{d}{d r} V_{1}(r)\right| \leq C r^{-4}, \quad\left|\frac{d}{d r}\left(\left(r^{2}+a^{2}\right)^{3} \frac{d}{d r} V_{1}(r)\right)\right| \leq C r .
$$

Therefore, $V$ cannot not have any critical points on $\left[r_{+}, r_{1}^{\prime}\right]$ and has a unique maximum $r_{\max } \in\left[r_{1}^{\prime}, \infty\right)$. The conclusion about the distance between $r_{\max }$ and $r_{\text {max }}^{0}$ is now equally straightforward.

We may now deduce the existence of an interval $\left(r_{\max }-\delta, r_{\max }+\delta\right)$ with the property that

$$
V(r)-\omega^{2} \geq c \Lambda, \quad \forall r \in\left(r_{\max }-\delta, r_{\max }+\delta\right)
$$

and

$$
-\left(r-r_{\max }\right) \frac{d}{d r} V(r) \geq c(\delta) \Lambda \frac{\left(r-r_{\max }\right)^{2}}{r^{4}}, \quad \forall r \in\left[r_{+}, \infty\right) .
$$

We now proceed to the construction of a suitable current for the regime $\mathcal{G} \sharp$. The current will be of the form:

$$
Q=Q_{0}^{f}+Q_{1}^{h}+\Gamma Q_{\mathrm{red}}^{z}-B Q_{T} .
$$

To elucidate the exposition we describe the procedure in stages.

1. We first apply current $Q_{0}^{f}$ with $f$ such that $f=-1$ at $r=r_{+}, f=0$ at $r=r_{\text {max }}$, $f=1$ at $r=\infty$ and $f^{\prime}(r)>0$ for all $r$, giving

$$
\begin{aligned}
\int_{r_{+}}^{\infty}\left[2 f^{\prime}\left|u^{\prime}\right|^{2}-f V^{\prime}|u|^{2}-\frac{1}{2} f^{\prime \prime \prime}|u|^{2}\right] & =\left[\left|u^{\prime}\right|^{2}+\left(\omega-\omega_{+}\right)^{2}|u|^{2}\right]_{r=r_{+}}+\left[\left|u^{\prime}\right|^{2}+\omega^{2}|u|^{2}\right]_{r=\infty} \\
& -\int_{r_{+}}^{\infty}\left[2 f \operatorname{Re}\left(u^{\prime} \bar{H}\right)+f^{\prime} \operatorname{Re}(u \bar{H})\right] .
\end{aligned}
$$

We impose additional conditions on the function $f$ to guarantee that the expression

$$
-f V^{\prime}-\frac{1}{2} f^{\prime \prime \prime} \geq 0
$$

for all values of $r \in\left[r_{+}, \infty\right)$. Since $f$ vanishes at $r=r_{\max }$ and $V^{\prime}$ obeys the property (73), we can easily arrange

$$
f V^{\prime} \geq c \Lambda \frac{\Delta\left(r-r_{\max }\right)^{2}}{r^{7}} .
$$

It remains to choose $f$ with the property that $f^{\prime \prime \prime}(r)<0$ in a small neighborhood of $r_{\max }$ and $\left|f^{\prime \prime \prime}(r)\right| \leq C \Delta r^{-5}$.

The left hand side of (74) is non-negative yet degenerate at $r=r_{\max }$. The condition $V\left(r_{\max }\right)-\omega^{2} \geq c \Lambda$ indicates this regime is non-trapped and the degeneracy may be removed with the help of the current $Q_{1}^{h}$. The problem however is a lack of control of the boundary terms on the right hand side due to the super-radiant condition. The latter will be handled with the help of the red-shift current $Q_{\text {red }}^{z}$. 
2. We now add a large multiple of the $Q_{1}^{h}$ current with a non-negative function $h$ supported in $\left[r_{\max }-\delta, r_{\max }+\delta\right]$ (that is in the region where $V(r)>\omega^{2}$ ), equal to one on $\left[r_{\max }-\delta / 2, r_{\max }+\delta / 2\right]$ :

$$
\begin{aligned}
\int_{r_{+}}^{\infty} & {\left[\left(2 f^{\prime}+A h\right)\left|u^{\prime}\right|^{2}+\left(A h\left(V-\omega^{2}\right)-f V^{\prime}\right)|u|^{2}-\frac{1}{2}\left(f^{\prime \prime \prime}+A h^{\prime \prime}\right)|u|^{2}\right] } \\
& =\left[\left|u^{\prime}\right|^{2}+\left(\omega-\omega_{+}\right)^{2}|u|^{2}\right]_{r=r_{+}}+\left[\left|u^{\prime}\right|^{2}+\omega^{2}|u|^{2}\right]_{r=\infty} \\
& -\int_{r_{+}}^{\infty}\left[2 f \operatorname{Re}\left(u^{\prime} \bar{H}\right)+\left(f^{\prime}+A h\right) \operatorname{Re}(u \bar{H})\right] .
\end{aligned}
$$

The left hand side of (75) is now positive and non-degenerate. Moreover, its integrand has the property that it is

$$
\geq A\left(\left|u^{\prime}\right|^{2}+\Lambda|u|^{2}\right), \quad \forall r \in\left[r_{\max }-\frac{\delta}{2}, r_{\max }+\frac{\delta}{2}\right] .
$$

The size of constant $A$ is limited only by the lower bound on the (large) frequency parameter $\Lambda$.

3. We now choose another large constant $\Gamma$ with the property that $\Gamma \ll A$ and add a $\Gamma$ multiple of the red-shift current $Q_{\text {red }}^{z}$ with $z=\Lambda \frac{\zeta}{\tilde{V}}$ and $\zeta=1$ on $\left[r_{+}, r_{\max }\right]$ and vanishing for $r>r_{\max }+\delta / 2$ :

$$
\begin{aligned}
\Gamma \int_{r_{+}}^{r_{\max }} \Lambda \frac{V^{\prime}}{\tilde{V}^{2}}\left|u^{\prime}+i\left(\omega-\omega_{+}\right) u\right|^{2}-\Gamma \int_{r_{\max }}^{r_{\max }+\delta / 2} \Lambda\left[\left(\frac{\zeta}{\tilde{V}}\right)^{\prime}\left|u^{\prime}+i\left(\omega-\omega_{+}\right) u\right|^{2}-\zeta^{\prime}|u|^{2}\right] \\
+\int_{r_{+}}^{\infty}\left[\left(2 f^{\prime}+A h\right)\left|u^{\prime}\right|^{2}+\left(A h\left(V-\omega^{2}\right)-f V^{\prime}\right)|u|^{2}-\frac{1}{2}\left(f^{\prime \prime \prime}+A h^{\prime \prime}\right)|u|^{2}\right] \\
=\left[\left|u^{\prime}\right|^{2}+\left(\left(\omega-\omega_{+}\right)^{2}-\Gamma \Lambda\right)|u|^{2}\right]_{r=r_{+}}+\left[\left|u^{\prime}\right|^{2}+\omega^{2}|u|^{2}\right]_{r=\infty} \\
-\int_{r_{+}}^{\infty}\left[2\left(f-\Lambda \frac{\zeta}{\tilde{V}}\right) \operatorname{Re}\left(u^{\prime} \bar{H}\right)+\left(f^{\prime}+A h\right) \operatorname{Re}(u \bar{H})\right] .
\end{aligned}
$$

The first term on the left hand side above is positive. To estimate the second term we note that we may assume that on $\left[r_{\max }, r_{\max }+\delta / 2\right]$, the potential $\tilde{V}$ has a lower bound $c \Lambda$. Therefore, its integrand can be estimated by

$$
\leq \Gamma C\left[\left|u^{\prime}\right|^{2}+\left(\omega^{2}+m^{2}\right)|u|^{2}\right] \ll A\left[\left|u^{\prime}\right|^{2}+\Lambda|u|^{2}\right],
$$

where the replacement of $\omega^{2}+m^{2}$ by $\Lambda$ is justified by the superradiant condition. Therefore the left hand side is positive definite. On the other hand, using the boundary conditions (54), the boundary term at $r=r_{+}$on the right hand side is

$$
\left(2\left(\omega-\omega_{+}\right)^{2}-\Gamma \Lambda\right)|u|^{2} \leq-\frac{\Gamma}{2} \Lambda|u|^{2}
$$

and thus now has a "good" sign and a large multiple constant. The other boundary term at $r=\infty$ still has the "wrong" sign but it is merely

$$
2 \omega^{2}|u|^{2}
$$

and therefore can be eliminated by a subtraction of a $B$ multiple of the $Q_{T}$ current

$$
\left.B \omega^{2}|u|^{2}\right|_{r=\infty}=-\left.B \omega\left(\omega-\omega_{+}\right)|u|^{2}\right|_{r=r_{+}}+B \omega \int_{r_{+}}^{\infty} \operatorname{Im}(u \bar{H}),
$$

which will create a bounded negative term at $r=r_{+}$. The latter in turn is easily dominated by $\frac{\Gamma}{2} \Lambda|u|^{2}$, provided that $B \ll \Gamma$. We can summarize the results obtained 
in this regime in the following inequality:

$$
\begin{aligned}
b\left(\int_{r_{+}}^{\infty} \frac{\Delta}{r^{4}}\right. & {\left.\left[\left|u^{\prime}\right|^{2}+\frac{\left(\omega^{2}+\Lambda\right)}{r}|u|^{2}\right]+\int_{r_{+}}^{r_{\max }} \frac{\left|u^{\prime}+i\left(\omega-\omega_{+}\right) u\right|^{2}}{r-r_{+}}+\left(\omega^{2}+\Lambda\right)\left[\left.|u|^{2}\right|_{r=r_{\max }}+\left.|u|^{2}\right|_{r=r_{\infty}}\right]\right) } \\
& =\int_{r_{+}}^{\infty}\left[-2\left(f-\Lambda \frac{\zeta}{\tilde{V}}\right) \operatorname{Re}\left(u^{\prime} \bar{H}\right)-\left(f^{\prime}+A h\right) \operatorname{Re}(u \bar{H})+B \omega \operatorname{Im}(u \bar{H})\right] .
\end{aligned}
$$

To end this section we observe that according to Remark 11.1 the frequency range $\mathcal{G}^{\sharp}$ can be slightly extended to

$$
0 \leq m \omega \leq \frac{a m^{2}}{2 M r_{+}}+\alpha \Lambda
$$

for some small constant $\Lambda$. This observation, in particular, will allow us to restrict the range $\mathcal{G}_{\text {দ }}$ to

$$
m \omega \notin\left[0, \frac{a m^{2}}{2 M r_{+}}+\alpha \Lambda\right]
$$

11.4. The $\mathcal{G}_{\sharp}$ range. This is a large frequency non-superradiant regime in which $\omega$ dominates all the other frequencies, that is $\Lambda$ and consequently $m$. Since this a non-superradiant range, the boundary terms at $r=r_{+}$and $r=\infty$ of all currents are dominated by the (positive definite) boundary terms of the $Q_{T}$ current.

In this regime, we shall apply a current

$$
Q=Q_{0}^{f}-Q_{1}^{h}-B Q_{T}
$$

with a monotonically increasing positive $f$ satisfying $1 \leq f \leq 2$, and the function $h=f^{\prime}$. We obtain

$$
\begin{aligned}
\int_{r_{+}}^{\infty} & {\left[f^{\prime}\left|u^{\prime}\right|^{2}+\left(f^{\prime}\left(\omega^{2}-V\right)-f V^{\prime}\right)|u|^{2}\right] } \\
& -\left[\left|u^{\prime}\right|^{2}-\left(\left(\omega-\omega_{+}\right)^{2}+B \omega\left(\omega-\omega_{+}\right)\right)|u|^{2}\right]_{r=r_{+}}-\left[\left|u^{\prime}\right|^{2}+(1-B) \omega^{2}|u|^{2}\right]_{r=\infty} \\
& -\int_{r_{+}}^{\infty}\left[2 f \operatorname{Re}\left(u^{\prime} \bar{H}\right)+B \omega \operatorname{Im}(u \bar{H})\right] .
\end{aligned}
$$

Given that $\omega^{2} \geq C \Lambda$ with a sufficiently large constant $C$ and

$$
|V| \leq C_{1} \Lambda+c_{1} \omega^{2}, \quad\left|V^{\prime}\right| \leq \frac{\Delta}{r^{5}}\left(C_{1} \Lambda+c_{1} \omega^{2}\right)
$$

for some universal constants (large $C_{1}$ and small $c_{1}$, which can be chosen to be arbitrarily small depending on th size of $C_{1}$ and a lower bound on $|\omega| \geq \omega_{1}$ )), we may choose $C$ so that the integrand on the left hand side is positive and dominates

$$
c_{1} f^{\prime}\left[\left|u^{\prime}\right|^{2}+\left(\omega^{2}+\Lambda\right)|u|^{2}\right] .
$$

The boundary terms are positive due to the boundary conditions $u^{\prime}=-i \omega\left(\omega-\omega_{+}\right)$ at $r=r_{+}$and $u^{\prime}=i \omega$ at $r=\infty$, the non-superradiance condition, and a choice of a sufficiently large constant $B$. Therefore, with an appropriate choice of $f$

$$
\begin{gathered}
b\left(\int_{r_{+}}^{\infty} \frac{\Delta}{r^{4}}\left[\left|u^{\prime}\right|^{2}+\left(\omega^{2}+\Lambda\right)|u|^{2}\right]+\left(\omega^{2}+\Lambda\right)\left[\left.|u|^{2}\right|_{r=r_{\max }}+\left.|u|^{2}\right|_{r=r_{\infty}}\right]\right) \\
\leq \int_{r_{+}}^{\infty}\left[-2 f \operatorname{Re}\left(u^{\prime} \bar{H}\right)+B \omega \operatorname{Im}(u \bar{H})\right] .
\end{gathered}
$$


11.5. The $\mathcal{G}_{\text {b }}$ range. This is a non-superradiant regime in which $\omega^{2}$ is comparable to $\Lambda$. This range corresponds to the trapping regime. For these frequencies potential $V_{0}$ may have at most two critical points $r_{\min }^{0}<r_{\max }^{0}$.

According to the Remark 11.1 and the discussion at the end of section 11.3 we may in fact assume that $m \omega \notin\left[0, \frac{a m^{2}}{2 M r_{+}}+\alpha \Lambda\right]$ for some small constant $\alpha$. Under this condition and since $\omega \sim \Lambda$,

$$
\omega^{2}-V\left(r_{+}\right)=\omega^{2}-V_{0}\left(r_{+}\right) \geq c \Lambda .
$$

We define $r_{0}$ to be the largest value with the property that for all $r \in\left[r_{+}, r_{0}\right)$

$$
V_{0}(r) \leq V_{0}\left(r_{+}\right)+\frac{c}{2} \Lambda \text {. }
$$

If $r_{0}$ is finite then, since $r_{\min }^{0}<r_{\max }^{0}$, it is clear that if $r_{\min }^{0}$ exists then

$$
r_{\min }^{0}<r_{0} \leq r_{\max }^{0} \text {. }
$$

Moreover, in that case, since $V_{0}(r) \rightarrow 0$ as $r \rightarrow \infty$, the potential $V_{0}$ does have a maximum point $r_{\max }^{0}$ and the value $r_{\max }^{0}$ is uniformly (in $\Lambda$ ) bounded from above with a bound dependent on $\lambda_{2}$. On the other hand, since $\left|\frac{d}{d r} V_{0}(r)\right| \leq C \Lambda r^{-3}$, the value $r_{0}$, and thus $r_{\max }^{0}$, is uniformly bounded away from $r_{+}$.

We now consider the value $r_{1}$, where the function

$$
\frac{d}{d r}\left[\left(r^{2}+a^{2}\right)^{3} \frac{d}{d r} V_{0}(r)\right]=-6 \Lambda\left[r^{2}-2 M r+4 M r \sigma+\frac{a^{2}}{3}-\frac{2}{3} a^{2} \frac{m^{2}}{\Lambda}\right],
$$

changes its sign from positive to negative. If $r_{1}$ belongs to the interval $\left[r_{+}, \infty\right)$, then $r_{1}<r_{\max }^{0}$. Moreover, since in this regime the parameter $\sigma=a m \omega / \Lambda$ is bounded, the value of $r_{1}$ is uniformly bounded from above. We consider two cases based on the value of $V_{0}\left(r_{1}\right)$.

If $V_{0}\left(r_{1}\right) \leq V_{0}\left(r_{+}\right)+\frac{3 c}{4} \Lambda$, then, in view of the fact that $V_{0}$ has a unique maximum at $r_{\max }^{0}$, we have that

$$
V_{0}(r) \leq V_{0}\left(r_{+}\right)+\frac{3 c}{4} \Lambda, \quad \forall r \in\left[r_{+}, \max \left(r_{0}, r_{1}\right)\right] .
$$

Moreover, we can find a small constant $\delta>0$ such that the above property holds on a slightly larger interval

$$
V_{0}(r) \leq V_{0}\left(r_{+}\right)+\frac{3 c}{4} \Lambda, \quad \forall r \in\left[r_{+}, \max \left(r_{0}, r_{1}\right)+\delta\right],
$$

and

$$
\frac{d}{d r}\left[\left(r^{2}+a^{2}\right)^{3} \frac{d}{d r} V_{0}(r)\right]<-c(\delta) \Lambda, \quad \forall r \in\left[\max \left(r_{0}, r_{1}\right)+\delta, \infty\right) .
$$

These conclusions are consequences of the simple bound $\left|\frac{d}{d r} V_{0}(r)\right| \leq C \Lambda r^{-3}$ and the fact that $\Lambda^{-1} \frac{d}{d r}\left[\left(r^{2}+a^{2}\right)^{3} \frac{d}{d r} V_{0}(r)\right]$ is a quadratic polynomial with bounded coefficients vanishing at the unique point $r_{1}$ on the interval $\left[r_{+}, \infty\right)$.

If, on the other hand, $V_{0}\left(r_{1}\right) \geq V_{0}\left(r_{+}\right)+\frac{3 c}{4} \Lambda$, then we have that $\left(r_{1}-r_{0}\right)$ is uniformly bounded from below by a small positive constant $c_{1}$, and since $\frac{d}{d r} V_{0}\left(r_{0}\right) \geq$ 0 , we can find a value $r_{0}^{\prime} \in\left[r_{0}, r_{1}\right]$ such that

$$
V_{0}(r) \leq V_{0}\left(r_{+}\right)+\frac{3 c}{4} \Lambda, \quad \forall r \in\left[r_{+}, r_{0}^{\prime}\right]
$$

and

$$
\frac{d}{d r} V_{0}(r) \geq c_{1} \Lambda, \quad \forall r \in\left[r_{0}^{\prime}, r_{1}\right] .
$$


Moreover, the last property can be easily extended to a slightly larger interval

$$
\frac{d}{d r} V_{0}(r) \geq c_{1} \Lambda, \quad \forall r \in\left[r_{0}^{\prime}, r_{1}+\delta\right]
$$

so that, in addition

$$
\frac{d}{d r}\left[\left(r^{2}+a^{2}\right)^{3} \frac{d}{d r} V_{0}(r)\right]<-c(\delta) \Lambda, \quad \forall r \in\left[r_{1}+\delta, \infty\right) .
$$

In both cases, we may therefore claim the existence of the value $r_{3}$, uniformly bounded away from $r_{+}$, such that

$$
V_{0}(r) \leq V_{0}\left(r_{+}\right)+\frac{3 c}{4} \Lambda \leq \omega^{2}-\frac{c}{4} \Lambda, \quad \forall r \in\left[r_{+}, r_{3}\right]
$$

and, such that, for any $r \in\left[r_{3}, \infty\right)$, either

or

$$
\frac{d}{d r} V_{0}(r) \geq c_{1} \Lambda
$$

$$
\frac{d}{d r}\left[\left(r^{2}+a^{2}\right)^{3} \frac{d}{d r} V_{0}(r)\right]<-c(\delta) \Lambda .
$$

We note that $r_{3}$ is either uniformly bounded from above or $r_{3}=\infty$.

Now adding a bounded potential $V_{1}$ we obtain that for $r \in\left[r_{+}, r_{3}\right]$

$$
V(r) \leq \omega^{2}-\frac{c}{4} \Lambda
$$

while on the interval $\left[r_{3}, \infty\right)$, if non-empty, potential $V$ has a unique non-degenerate maximum at $r_{\max }, \Lambda^{-1}$-close to $r_{\max }^{0}$, and

$$
\frac{d^{2}}{d r^{2}} V\left(r_{\max }\right)<-c_{1} \Lambda, \quad \frac{d}{d r} V\left(r_{3}\right)>c_{1} \Lambda .
$$

In this regime we use a combination

$$
Q=Q_{0}^{f}-Q_{2}^{y}-B Q_{T}
$$

of the currents $Q_{0}, Q_{2}$ and $Q_{T}$. The current $Q_{0}^{f}$ is applied with a function $f$ which vanishes on the horizon, monotonically increases on $\left[r_{3}, \infty\right)$, changes its sign from negative to positive at $r=r_{\max }$ and approaches 1 as $r \rightarrow \infty$, and satisfies the additional condition that

$$
f^{\prime \prime \prime}(r)<0, \quad \forall r \in\left[r_{3}, \infty\right)
$$

The second current will be $Q_{2}^{y}$, restricted to the interval $\left[r_{+}, r_{3}\right]$, with a monotonically decreasing function $y$. Finally, we subtract a large multiple of the current $Q_{t}$. We obtain for $Q=Q_{0}^{f}-Q_{2}^{y}-B Q_{T}$ :

$$
\begin{aligned}
-\int_{r_{+}}^{r_{3}}\left[y^{\prime}\left(\left|u^{\prime}\right|^{2}+\left(\omega^{2}-V\right)|u|^{2}\right)-y V^{\prime}|u|^{2}\right]+\int_{r_{+}}^{\infty}\left[2 f^{\prime}\left|u^{\prime}\right|^{2}-\left(f V^{\prime}+\frac{1}{2} f^{\prime \prime \prime}\right)|u|^{2}\right] \\
+\left[-f\left|u^{\prime}\right|^{2}+(B-f) \omega^{2}|u|^{2}\right]_{r=\infty}+\left.\left[B \omega\left(\omega-\omega_{+}\right)-2 y\left(\omega-\omega_{+}\right)^{2}\right]|u|^{2}\right|_{r=r_{+}} \\
=-\int_{r_{+}}^{\infty}\left[2 f \operatorname{Re}\left(u^{\prime} \bar{H}\right)-f^{\prime} \operatorname{Re}(u \bar{H})-B \omega \operatorname{Im}(u \bar{H})\right]+\int_{r_{+}}^{r_{3}}\left[y \operatorname{Re}\left(u^{\prime} \bar{H}\right)\right] .
\end{aligned}
$$

By the described properties of the potential $V$, the expression $-\left(f V^{\prime}+\frac{1}{2} f^{\prime \prime \prime}\right)$ is positive on the interval $\left[r_{3}, \infty\right)$. On the interval $\left[r_{+}, r_{3}\right]$, we choose a function $y$ so that

$$
-y^{\prime}\left(\omega^{2}-V\right)+y V^{\prime}-\left(f V^{\prime}+\frac{1}{2} f^{\prime \prime \prime}\right) \geq 0
$$


Since for these values of $r$

$$
\left(\omega^{2}-V\right) \geq c_{1} \Lambda, \quad\left|V^{\prime}\right| \leq C \Lambda \frac{\Delta}{r^{2}}, \quad|f|+\left|f^{\prime \prime \prime}\right| \leq C \frac{\Delta}{r^{2}}
$$

for some positive constants $c_{1}, C$, it suffices to to fulfill the inequality

$$
-\frac{d}{d r} y \geq y C+C
$$

provided that the constructed function $y$ is positive and monotonically decreasing. The function

$$
y=-C e^{-C r} \int_{r}^{r_{3}} e^{C r^{\prime}} d r^{\prime}=1-e^{C\left(r-r_{3}\right)}
$$

satisfies all the above criteria. Finally, a choice of a large constant positive constant $B$ together with the non super-radiant condition $m \omega \notin\left[0, m \omega_{+}\right]$and the boundary condition $u^{\prime}=i \omega u$ at $r=\infty$ ensure that both boundary terms at $r=r_{+}$and $r=\infty$ are positive.

We summarise the results of this regime with the following inequality, which holds under appropriate assumptions on $f$ :

$$
\begin{aligned}
b\left(\int_{r_{+}}^{\infty} \frac{\Delta}{r^{4}}\right. & {\left.\left[\left|u^{\prime}\right|^{2}+\left(\omega^{2}+\Lambda\right) \frac{\left(r-r_{\max }\right)^{2}}{r^{3}}|u|^{2}\right]+\left(\omega^{2}+\Lambda\right)\left[\left.|u|^{2}\right|_{r=r_{\max }}+\left.|u|^{2}\right|_{r=r_{\infty}}\right]\right) } \\
\leq & \int_{r_{+}}^{\infty}\left[-2 f \operatorname{Re}\left(u^{\prime} \bar{H}\right)+f^{\prime} \operatorname{Re}(u \bar{H})+B \omega \operatorname{Im}(u \bar{H})\right]+\int_{r_{+}}^{r_{3}}\left[y \operatorname{Re}\left(u^{\prime} \bar{H}\right)\right] .
\end{aligned}
$$

11.6. The $\mathcal{G}_{\hbar}$ range. This is a non-superradiant regime in which the frequency $\omega^{2}$ is much smaller than $\Lambda$. In particular, by Remark 11.1, the potential $V_{0}$ has only one critical point-a maximum-at the value $r=r_{\text {max }}^{0}$. Repeating the arguments which we applied to the regime $\mathcal{G}^{\sharp}$ we also conclude that the potential $V$ has a unique non-degenerate critical maximal point at $r_{\max }$, which is uniformly bounded away from $r_{+}$and is bounded from above. Similarly, we also have an interval $\left(r_{\max }-\delta, r_{\max }+\delta\right)$ with the property that

$$
V(r)-\omega^{2} \geq c \Lambda, \quad \forall r \in\left(r_{\max }-\delta, r_{\max }+\delta\right)
$$

and

$$
\left(r-r_{\max }\right) \frac{d}{d r} V(r) \geq c \Lambda \frac{\left(r-r_{\max }\right)^{2}}{r^{4}}, \quad \forall r \in\left(r_{+}, \infty\right) .
$$

We repeat the construction of $\mathcal{G}^{\sharp}$ with the current $Q=Q_{0}^{f}+A Q_{1}^{h}$ and the same choice of the functions $f$ and $h$, which gives

$$
\begin{aligned}
\int_{r_{+}}^{\infty} & {\left[\left(2 f^{\prime}+A h\right)\left|u^{\prime}\right|^{2}+\left(A h\left(V-\omega^{2}\right)-f V^{\prime}\right)|u|^{2}-\frac{1}{2}\left(f^{\prime \prime \prime}+A h^{\prime \prime}\right)|u|^{2}\right] } \\
& =\left[\left|u^{\prime}\right|^{2}+\left(\omega-\omega_{+}\right)^{2}|u|^{2}\right]_{r=r_{+}}+\left[\left|u^{\prime}\right|^{2}+\omega^{2}|u|^{2}\right]_{r=\infty} \\
& -\int_{r_{+}}^{\infty}\left[2 f \operatorname{Re}\left(u^{\prime} \bar{H}\right)+\left(f^{\prime}+A h\right) \operatorname{Re}(u \bar{H})\right],
\end{aligned}
$$

with a non-degenerate positive definite integrand on the left hand side. In this case however, due to the non super-radiance condition, we may simply subtract a large 
multiple of the current $Q_{T}$ to handle the boundary terms

$$
\begin{aligned}
\int_{r_{+}}^{\infty} & {\left[\left(2 f^{\prime}+A h\right)\left|u^{\prime}\right|^{2}+\left(A h\left(V-\omega^{2}\right)-f V^{\prime}\right)|u|^{2}-\frac{1}{2}\left(f^{\prime \prime \prime}+A h^{\prime \prime}\right)|u|^{2}\right] } \\
& +B \omega\left(\omega-\omega_{+}\right)|u|_{r=r_{+}}^{2}+\left.B \omega^{2}|u|^{2}\right|_{r=\infty} \\
& =\left[\left|u^{\prime}\right|^{2}+\left(\omega-\omega_{+}\right)^{2}|u|^{2}\right]_{r=r_{+}}+\left[\left|u^{\prime}\right|^{2}+\omega^{2}|u|^{2}\right]_{r=\infty} \\
& -\int_{r_{+}}^{\infty}\left[2 f \operatorname{Re}\left(u^{\prime} \bar{H}\right)+\left(f^{\prime}+A h\right) \operatorname{Re}(u \bar{H})-B \operatorname{Im}(u \bar{H})\right] .
\end{aligned}
$$

We summarise:

$$
\begin{aligned}
b\left(\int_{r_{+}}^{\infty} \frac{\Delta}{r^{4}}\right. & {\left.\left[\left|u^{\prime}\right|^{2}+\frac{\left(\omega^{2}+\Lambda\right)}{r}|u|^{2}\right]+\left(\omega^{2}+\Lambda\right)\left[\left.|u|^{2}\right|_{r=r_{\max }}+\left.|u|^{2}\right|_{r=r_{\infty}}\right]\right) } \\
& \leq \int_{r_{+}}^{\infty}\left[-2 f \operatorname{Re}\left(u^{\prime} \bar{H}\right)-\left(f^{\prime}+A h\right) \operatorname{Re}(u \bar{H})+B \omega \operatorname{Im}(u \bar{H})\right] .
\end{aligned}
$$

11.7. The $\mathcal{G}_{b}$ range. This is a regime in which all frequencies $\omega, m$ and $\lambda_{m \ell}$ are bounded. Recall that in the small $a$ case, a general construction for such frequencies was presented in Section 10.2 of this paper. As shown in [18], this construction also holds for general $a<M$ in the case $m=0$. Moreover, it can be easily adapted to cover more generally non-superradiant modes in this regime. To handle the remaining bounded superradiant frequencies in this regime, the argument requires a modification of Whiting's results on the absence of exponentially growing modes in [47]. The details will be given in [22].

We note that since the azimuthal frequency $m$ can be defined without the Fourier transform in time, we can define a priori the projection $P_{m}$ to the $m^{\prime}$ th azimuthal mode. Clearly, solutions of the wave equation such that $P_{m} \psi=0$ for $1 \leq|m| \leq m_{0}$ and large $m_{0}$ are not supported at all in the frequency range $\mathcal{G}_{b}$. Thus, for such solutions, this appeal to [47] is unnecessary and we have given here a completely self-contained discussion of all constructions necessary for the proof.

11.8. Summation and the local integrated energy decay. We have thus obtained the inequalities (61) for all frequencies, as required, and we may thus apply Section 9.3.3 to obtain

$$
b \int_{0}^{\infty} d \tau \int_{\Sigma_{\tau} \cap\left\{r \leq r_{+}\right\}}\left(\chi|\partial \psi|^{2}+\left|\partial_{r} \psi\right|^{2}+|\psi|^{2}\right) \leq \int_{\Sigma_{0}}|\partial \psi|^{2} .
$$

The precise form of the estimates of Section 11.5 now reveals the nature of the physical-space degeneration function $\chi$ : The function $\chi$ must vanish in a neighborhood of the projection of the 'trapped set'

$$
\left\{r: r \in \cap_{L=1}^{\infty}\left(\cup_{\ell \geq L} r_{m \ell}^{a \omega}\right)\right\},
$$

where $r_{m \ell}^{a \omega}$ are the maximum values $r_{\max }$ of the potential $V$ in the $\mathcal{G}_{\text {b }}$ range.

Of course, we have really obtained a stronger estimate than (76), namely, we obtain the boundedness of the sum of the left hand sides of the versions of (61) obtained here, where each microlocalised $\chi$ degenerates at most at a single point. The degeneration of this stronger estimate however, as opposed to (76), can thus only be understood microlocally.

The reader should return to Section 9.3.3 to obtain the remaining statements of Theorem 1. 


\section{REFERENCES}

[1] S. Alexakis, A. Ionescu and S. Klainerman Uniqueness of smooth stationary black holes in vacuum: small perturbations of the Kerr spaces, arXiv:0904.0982

[2] S. Alinhac Energy multipliers for perturbations of Schwarzschild metric, Comm. Math. Phys. 288 (2009), no. 1, 199-224

[3] L. Andersson and P. Blue Hidden symmetries and decay for the wave equation on the Kerr spacetime, arXiv:0908.2265

[4] S. Aretakis The wave equation on extreme Reissner-Nordström black hole spacetimes: stability and instability results, arXiv:1006.0283

[5] P. Blue Decay of the Maxwell field on the Schwarzschild manifold, J. Hyperbolic Differ. Equ. 5 (2008), no. 4, 807-856

[6] P. Blue and A. Soffer Semilinear wave equations on the Schwarzschild manifold. I. Local decay estimates, Adv. Differential Equations 8 (2003), no. 5, 595-614

[7] P. Blue and A. Soffer Errata for "Global existence and scattering for the nonlinear Schrodinger equation on Schwarzschild manifolds", "Semilinear wave equations on the Schwarzschild manifold I: Local Decay Estimates", and "The wave equation on the Schwarzschild metric II: Local Decay for the spin 2 Regge Wheeler equation", gr-qc/0608073

[8] P. Blue and J. Sterbenz Uniform decay of local energy and the semi-linear wave equation on Schwarzschild space, Comm. Math. Phys. 268 (2006), no. 2, 481-504

[9] B. Carter Global structure of the Kerr family of gravitational fields Phys. Rev. 174 (1968), 1559-1571

[10] B. Carter Hamilton-Jacobi and Schrödinger separable solutions of Einstein's equations, Comm. Math. Phys. 10 (1968), 280-310

[11] S. Chandrasekhar The mathematical theory of black holes, Oxford University Press, 1983.

[12] D. Christodoulou and S. Klainerman Asymptotic properties of linear field equations in Minkowski space, Comm. Pure Appl. Math. 43 (1990), no. 2, 137-199.

[13] D. Christodoulou and S. Klainerman The global nonlinear stability of the Minkowski space, Princeton University Press, 1993

[14] M. Dafermos and I. Rodnianski The redshift effect and radiation decay on black hole spacetimes Comm. Pure Appl. Math. 52 (2009), 859-919

[15] M. Dafermos and I. Rodnianski The wave equation on Schwarzschild-de Sitter spacetimes, arXiv:0709.2766v1 [gr-qc]

[16] M. Dafermos and I. Rodnianski A note on energy currents and decay for the wave equation on a Schwarzschild background, arXiv:0710.0171v1 [math.AP]

[17] M. Dafermos and I. Rodnianski A proof of the uniform boundedness of solutions to the wave equation on slowly rotating Kerr backgrounds, arXiv:0805.4309v1 [gr-qc]

[18] M. Dafermos and I. Rodnianski Decay for solutions of the wave equation on Kerr exterior spacetimes I-II: The cases $|a| \ll M$ or axisymmetry, preprint

[19] M. Dafermos and I. Rodnianski Lectures on black holes and linear waves, arXiv:0811.0354 [gr-qc]

[20] M. Dafermos and I. Rodnianski A new physical-space approach to decay for the wave equation with applications to black hole spacetimes, in XVIth International Congress on Mathematical Physics, P. Exner (ed.), World Scientific, London, 2009, arXiv:0910.4957v1 [math.AP]

[21] M. Dafermos and I. Rodnianski Decay for solutions of the wave equation on Kerr exterior spacetimes III: The case $|a|<M$, in preparation

[22] M. Dafermos and I. Rodnianski, forthcoming

[23] F. Finster, N. Kamran, J. Smoller, S. T. Yau Decay of solutions of the wave equation in Kerr geometry, Comm. Math. Phys. 264 (2006), 465-503

[24] F. Finster, N. Kamran, J. Smoller, S.-T. Yau Erratum: Decay of solutions of the wave equation in Kerr geometry, Comm. Math. Phys., online first

[25] J. B. Hartle and D. C. Wilkins Analytic properties of the Teukolsky equation, Commun. Math. Phys. 38 (1974), 47-63

[26] G. Holzegel, Ultimately Schwarzschildean spacetimes and the black hole stability problem, arXiv: 1010.3216

[27] B. Kay and R. Wald Linear stability of Schwarzschild under perturbations which are nonvanishing on the bifurcation 2-sphere, Classical Quantum Gravity 4 (1987), no. 4, 893-898 
[28] R. Kerr Gravitational field of a spinning mass as an example of algebraically special metrics, Phys. Rev. Lett. 11 (1963) 237-238

[29] I. Laba and A. Soffer Global existence and scattering for the nonlinear Schrödinger equation on Schwarzschild manifolds, Helv. Phys. Acta 72 (1999), no. 4, 272-294

[30] W. Littman The wave operator and $L_{p}$ norms, J. Math. Mech. 12 (1963), 55-68

[31] J. Luk Improved decay for solutions to the linear wave equation on a Schwarzschild black hole, Ann. Henri Poincaré, Online first, 2010, arXiv:0906.5588

[32] J. Luk A Vector Field Method Approach to Improved Decay for Solutions to the Wave Equation on a Slowly Rotating Kerr Black Hole, arXiv:1009.0671

[33] H. Lindblad and I. Rodnianski Global stability of Minkowski space-time in harmonic gauge, Ann. Math. 171 (2010), No. 3, 1401-1477

[34] J. Marzuola, J. Metcalfe, D. Tataru, M. Tohaneanu Strichartz estimates on Schwarzschild black hole backgrounds, Comm. Math. Phys. 293, (2010), 37-83

[35] J. R. Oppenheimer and H. Snyder On continued gravitational contraction, Phys. Rev. 56 (1939), 455-459

[36] W. H. Press and S. A. Teukolsky Perturbations of a rotating black hole. II. Dynamical stability of the Kerr metric, The Astrophysical Journal 185 (1973), 649-673

[37] J. Ralston Solutions of the wave equation with localized energy, Comm. Pure Appl. Math. 22 (1969), 807-823

[38] T. Regge and J. Wheeler Stability of a Schwarzschild singularity, Phys. Rev. 108 (1957), 10631069.

[39] A. Starobinsky Amplification of waves during reflection from a black hole, Soviet Physics JETP 37 (1973), 28-32

[40] D. Tataru Local decay of waves on asymptotically flat stationary space-times, arXiv:0910.5290

[41] D. Tataru and M. Tohaneanu Local energy estimate on Kerr black hole backgrounds, to appear in IMRN, 2010

[42] M. Tohaneanu Strichartz estimates on Kerr black hole backgrounds, arXiv:0910.1545

[43] S. Teukolsky Perturbations of a Rotating Black Hole. I. Fundamental Equations for Gravitational, Electromagnetic, and Neutrino-Field Perturbations, Astroph. J. 185 (1973), 635-648.

[44] M. Walker and R. Penrose On quadratic first integrals of the geodesic equations for type 22 spacetimes Comm. Math. Phys. 18 (1970), 265-274.

[45] C.V. Vishveshwara Stability of the Schwarzschild metric, Phys. Rev. D 1 (1970), 2870-2879.

[46] R. M. Wald Note on the stability of the Schwarzschild metric, J. Math. Phys. 20 (1979), 1056-1058

[47] B. Whiting Mode stability of the Kerr black hole, J. Math. Phys. 30 (1989), 1301

[48] S. Yang Global solutions to nonlinear wave equations in time dependent inhomogeneous media, arXiv:1010.4341

[49] Y. Zeldovich The Generation of Waves by a Rotating Body, Pisma v Redakstiyu Zhurnal Eksperimentalnoi i Teoreticheskoi Fiziki 14, (1971), 270

University of Cambridge, Department of Pure Mathematics and Mathematical Statistics, Wilberforce Road, Cambridge CB3 0WB United Kingdom

Princeton University, Department of Mathematics, Fine Hall, Washington Road, Princeton, NJ 08544 United States 